\title{
Relative Continuous-Time SLAM
}

\author{
Sean Anderson, Kirk MacTavish, and Timothy D. Barfoot
}

Version Pre-print

Citation Anderson S, MacTavish K, and Barfoot T D. "Relative Continuous-

(published version) Time SLAM”. International Journal of Robotics Research, 34(12):1453$1479,2015$.

How to cite TSpace items

Always cite the published version, so the author(s) will receive recognition through services that track citation counts, e.g. Scopus. If you need to cite the page number of the author manuscript from TSpace because you cannot access the published version, then cite the TSpace version in addition to the published version using the permanent URI (handle) found on the record page.

This article was made openly accessible by $U$ of $T$ Faculty. Please tell us how this access benefits you. Your story matters. 


\title{
Relative Continuous-Time SLAM
}

\author{
Sean Anderson, Kirk MacTavish, and Timothy D. Barfoot \\ Autonomous Space Robotics Lab, University of Toronto Institute for Aerospace Studies \\ \{sean.anderson,kirk.mactavish\}@mail.utoronto.ca, tim.barfooteutoronto.ca
}

\begin{abstract}
Appearance-based techniques for simultaneous localization and mapping (SLAM) have been highly successful in assisting robot motion estimation. However, these vision-based technologies have long assumed the use of imaging sensors with a global shutter, which are well suited to the traditional, discrete-time formulation of visual problems. In order to adapt these technologies to use scanning sensors, we propose novel methods for both outlier rejection and batch nonlinear estimation. Traditionally, the SLAM problem has been formulated in a single privileged coordinate frame, which can become computationally expensive over long distances, particularly when a loop closure requires the adjustment of many pose variables. Recent discrete-time estimators have shown that a completely relative coordinate framework can be used to incrementally find a close approximation of the full maximum-likelihood solution in constant time. In order to use scanning sensors, we propose moving the relative coordinate formulation of SLAM into continuous time by estimating the velocity profile of the robot. We derive the relative formulation of the continuous-time robot trajectory and formulate an estimator using temporal basis functions. A motion-compensated outlier rejection scheme is proposed by using a constant-velocity model for the random sample consensus (RANSAC) algorithm. Our experimental results use intensity imagery from a two-axis scanning lidar; due to the sensors' scanning nature, it behaves similarly to a slow rolling-shutter camera. Both algorithms are validated using a sequence of 6880 lidar frames acquired over a $1.1 \mathrm{~km}$ traversal.
\end{abstract}

\section{Introduction}

Visual odometry (VO) is a powerful 6D motion-estimation technique that is made efficient by temporally tracking points of interest, or appearance-based features, over a sequence of images. This technique is widely used due to the low cost and availability of passive camera technology. Due to the effectiveness of visual algorithms, much recent effort has been focused on what is known as the full SLAM, or bundle adjustment, problem. The objective of this problem is to minimize the reprojection error of many temporally matched appearance-based features by parameterizing and optimizing both the 3D

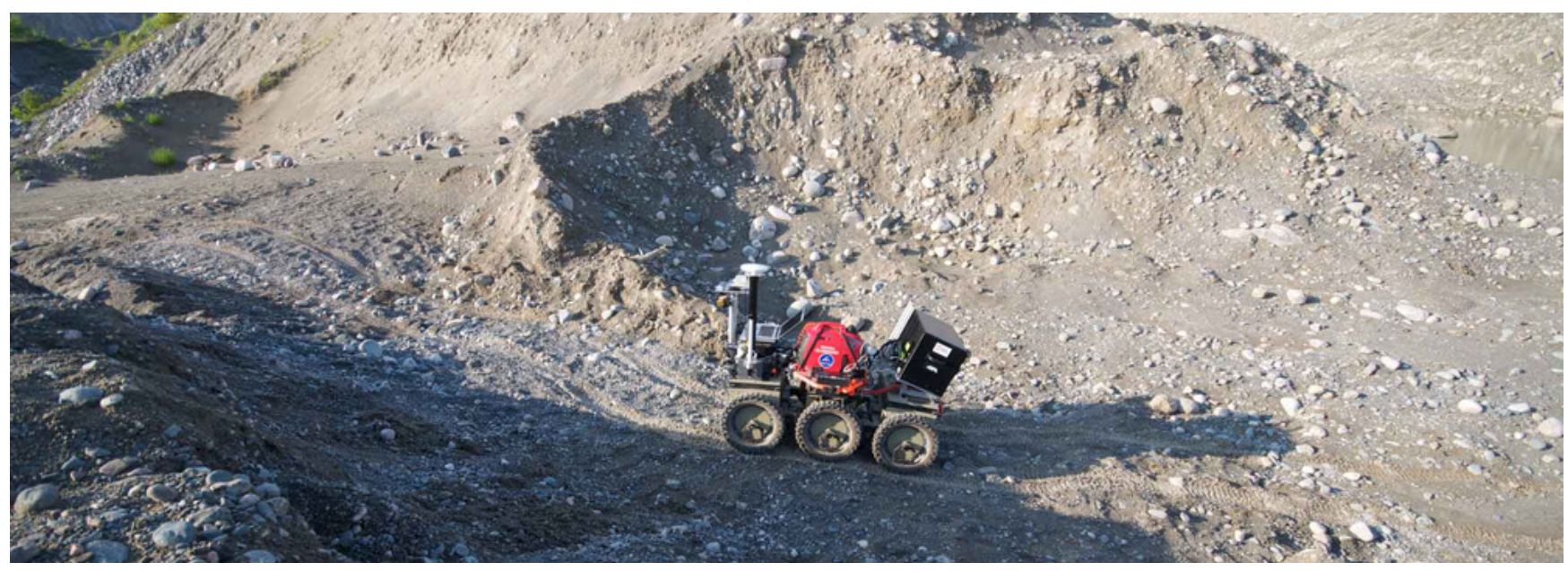

Figure 1: The ROC6 at the Ethier Sand and Gravel pit in Sudbury, Ontario, Canada. The large black instrument mounted on the front of the robot is an Autonosys scanning lidar that we use for scanning-while-moving motion estimation. 


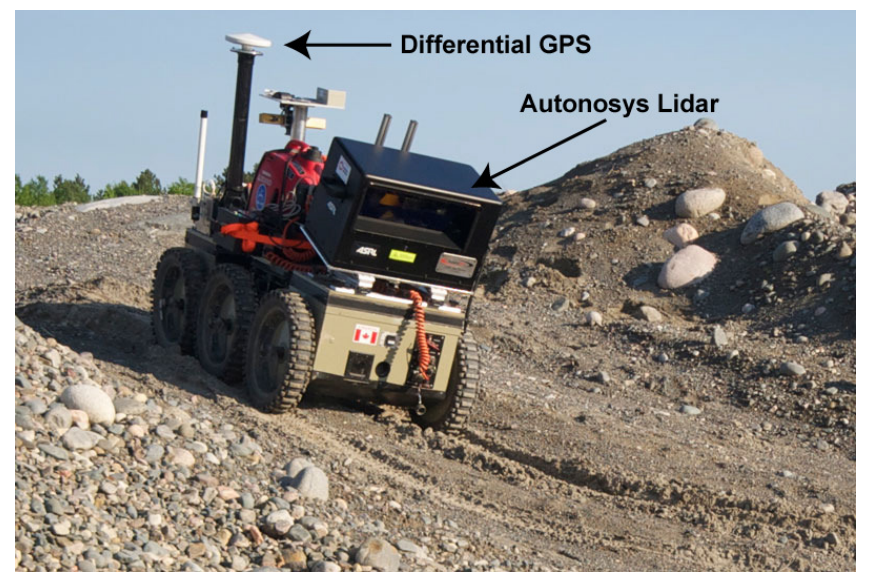

Figure 2: The ROC6 rover, equipped with an Autonosys LVC0702 lidar and a Thales DG-16 Differential GPS unit.

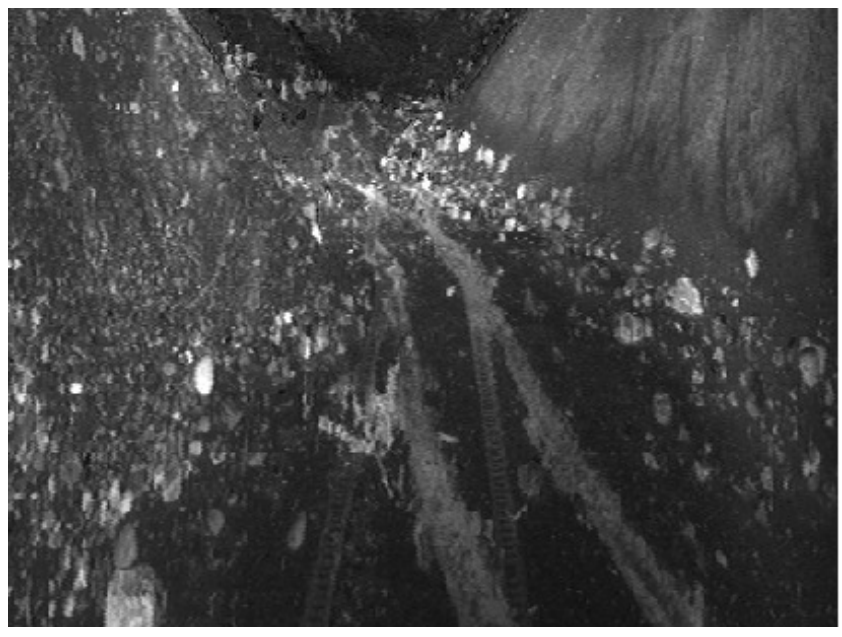

(a) Intensity Image

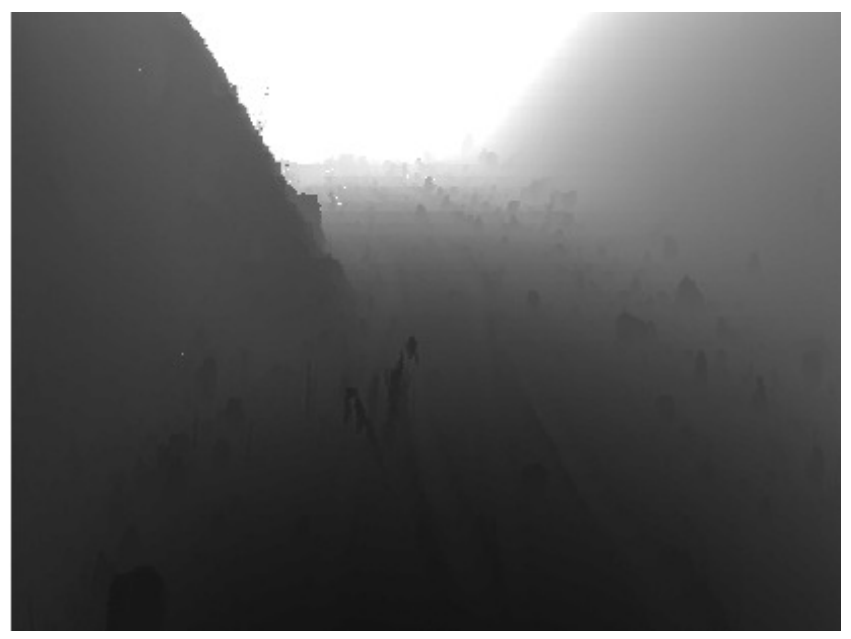

(b) Range Image

Figure 3: Corresponding intensity and range images formed using data from the Autonosys LVC0702 and the method developed by McManus et al. (2011). Intensity imagery is very robust to changes in lighting conditions; using this technology, even in complete darkness, we are able to perform appearance-based feature extraction and tracking.

feature positions and the 6D sensor pose at each image acquisition time.

SLAM is considered a core competency for autonomous mobile robots (Thrun et al., 2005) because it is often a prerequisite to path planning, navigation, and manipulation tasks. Traditionally, SLAM techniques are applied in a single privileged coordinate frame, which can become computationally expensive over long distances, particularly when a loop closure requires the adjustment of many pose variables. A recent approach to SLAM, and bundle adjustment, advocates that a single privileged coordinate frame is not a requirement to accomplish many of the common robotic tasks (Sibley et al., 2009). By reformulating the optimization problem to use relative coordinates, an incremental update strategy can be used to perform SLAM in constant time, even at loop closure.

Most of the visual SLAM and bundle adjustment algorithms have only been validated with the use of passive camera technology, which causes them to rely on consistent ambient lighting in order to find temporally similar appearance-based features. In a real-world scenario, such as the test environment shown in Figure 1, appearance can differ drastically with changes in lighting conditions; for example, change in the orientation of shadows, or more severely, the total absence of light.

In order to enable robust estimation under varying lighting conditions, active sensors such as lidar are an attractive alternative. 


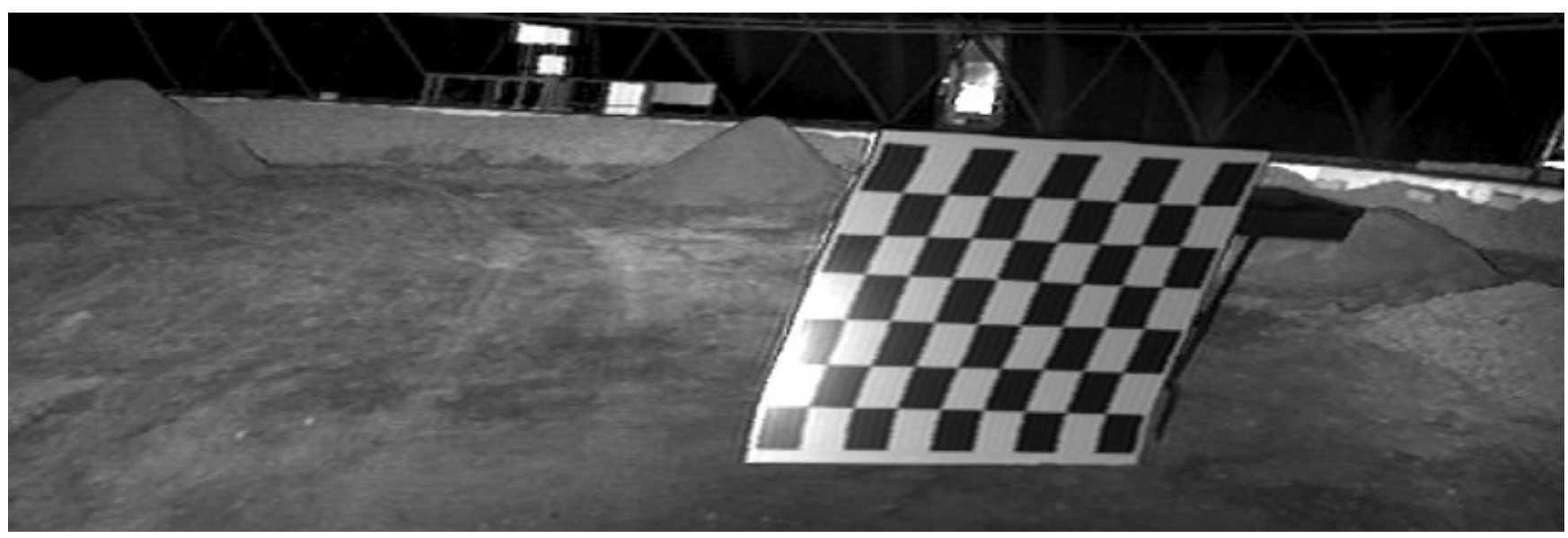

Figure 4: This figure shows an example intensity image captured using the Autonosys LVC0702 two-axis scanning lidar. The nonaffine image distortion is caused by a yaw-type motion during image acquisition. Note the irregular deformation of the square checkerboard pattern.

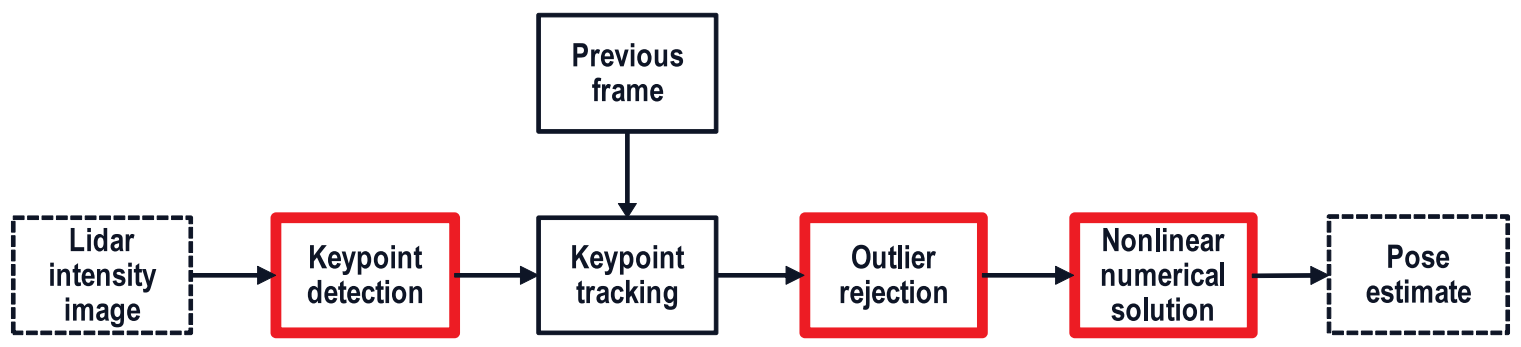

Figure 5: The typical visual pipeline used for visual odometry using lidar intensity images (McManus et al., 2013). Beginning with a new image, keypoints, or appearance-based features, are extracted from the image and matched against keypoints extracted from the previous image. Outlier rejection, typically a RANSAC algorithm, is then used to qualify a likely set of inliers from the proposed keypoint correspondences. Finally, a nonlinear numerical solution is used to produce a pose estimate. We bring attention to the fact that this pipeline is likely affected by motion distortion in the three highlighted (in red) modules: keypoint detection (due to nonaffine image distortion), outlier rejection (due to assumptions made by traditional RANSAC models), and the nonlinear numerical solution (due to the discrete nature of traditional pose estimation).

Specifically, we investigate the use of an Autonosys LVC0702, a two-axis scanning lidar, as seen on our robot in Figure 2. Although it is possible to use dense lidar data for SLAM, this research is particularly interested in exploiting the visual techniques that rely on sparse feature measurements. We use the method developed by McManus et al. (2011) to form the lidar intensity and range data into images, as seen in Figure 3. We extract speeded-up robust features (SURF) (Bay et al., 2006) from the intensity images that can be matched, independent of lighting conditions; this tactic allows us to perform visual odometry even in complete darkness (Barfoot et al., 2013).

In contrast to a charge-coupled device (CCD) that has a global shutter, the slow vertical scan of the Autonosys causes nonaffine image distortion, as seen in Figure 4, based on the velocity of the robot and the capture rate of the sensor. Similar in nature to cameras using complementary metal-oxide-semiconductor (CMOS) technology, which are known to be cheaper than ones using a CCD, these sensors are often avoided in robotics due to the added complexity of rolling-shutter-type distortions.

In the presence of motion-distorted imagery, there are three places in which the traditional visual pipeline is affected, shown in Figure 5. Due to nonaffine image distortions, the matching performance of typical feature detectors and descriptors (including SURF) begins to degrade when the sensor acquisition rate is inadequate for the platform speed. For our particular setup, with a platform speed of up to $0.5 \mathrm{~m} / \mathrm{s}$, and an Autonosys frame rate of $2 \mathrm{~Hz}$, we found that SURF still performs sufficiently well. Outside the scope of this paper, a separate study is being conducted on the use of alternative feature detectors and descriptors for motion-distorted imagery. 
Before considering a motion-estimation problem that properly accounts for this type of data, we must first confront the issue of feature correspondence and outlier rejection. An initial guess of the temporal tracks between two sets of features is often generated using appearance-based feature descriptors; this alone does not take into account the geometry of the problem and is prone to generating mismatches. The standard outlier-rejection scheme for filtering the initial guess is random sample consensus (RANSAC), which is a nondeterministic algorithm for robustly finding the parameters of a mathematical model that best describe a likely set of inliers (Fischler and Bolles, 1981). The model used in the RANSAC algorithm for the globalshutter, visual pipeline is a single rigid transformation (i.e., translation and rotation). In order to account for the temporal nature of rolling-shutter-type images, a RANSAC algorithm using a 6D constant-velocity model is proposed.

Finally, discrete-time batch estimation techniques typically require a pose variable for each measurement with a unique timestamp. Therefore, sensors that provide high-rate asynchronous measurements cause the state size to become unmanageable; furthermore, in the case of visual measurements with unique timestamps, the optimization problem becomes inadequately constrained without a motion model or odometry-type measurements to correlate the poses.

Continuous-time batch estimation is a recent strain of research that aims to address some of the issues with typical discrete-time estimation schemes; namely, the use of high-rate, motion-distorted, or unsynchronized sensors. Using a continuous trajectory model allows for an effective means to include high rate data, such as inertial measurement unit (IMU) measurements, or individually timestamped visual feature measurements, without negatively impacting state size. In order to create an estimation framework that is capable of incrementally building the full SLAM solution in constant time, despite the use of high-rate sensors, we propose moving the relative formulation of SLAM (Sibley et al., 2009) into continuous time.

This paper builds on the work presented in two of our recent conference papers (Anderson and Barfoot, 2013a,b) and extends them by considering large-scale loop closures. Using the place-recognition algorithm in MacTavish and Barfoot (2014), loop closures are detected and included in the window-filter-style optimizations conducted by the relative continuous-time SLAM algorithm. We also extend the conference papers by investigating the conditions under which the proposed outlier rejection schemes in Anderson and Barfoot (2013b) are solvable.

The remainder of this paper is organized as follows: in Section 2 we begin with a review of related work, in Section 3 we derive the extension of continuous-time batch estimation to use relative coordinates and propose a parametric solution using temporal basis functions that can be solved in constant-time (even at loop closure), in Section 4 we derive both a general nonlinear and point-based RANSAC algorithm for motion-distorted 3D visual sensors using a constant-velocity model, in Section 5 both the outlier rejection and nonlinear estimation schemes are validated using a 6880 frame sequence of lidar data captured over a $1.1 \mathrm{~km}$ trajectory, and in Section 6 we provide concluding remarks and discussion.

\section{Related Work}

The contents of this section will discuss prior art relevant to both the nonlinear state estimator and the outlier rejection scheme proposed in this paper; a focus will be placed on techniques that aim to solve similar problems using motion-distorted data.

Performing bundle adjustment using sparse visual feature sets is a problem with foundations from over half a century ago (Brown, 1958). Throughout the years, estimators have focused on becoming faster and more suitable for mapping over great distances; formulating the full nonlinear least-squares optimization problem has remained a dominant paradigm because it is both flexible and efficient (Triggs et al., 2000). Exploiting the use of a graph structure has been fruitful for many recent batch estimation algorithms (Sibley et al., 2009; Dellaert and Kaess, 2006; Konolige et al., 2010); the completely relative formulation of the bundle adjustment problem, by Sibley et al. (2009), is of particular relevance to this work. In order to run in constant time, the technique incrementally updates an adaptive region of state variables that are anticipated to change in light of new information. More importantly, the graph of relative pose changes allows for constant-time operation during loop closure. Similarly to the approach using a kinematic chain of relative pose estimates, our method uses a continuous-time estimate of the robot's velocity profile; this profile can be thought of as a kinematic chain with an infinite number of relative pose changes.

It is well known that traditional batch estimation techniques struggle when using ranging or imaging sensors that are affected by motion. In many cases, the effects of motion distortion are usually ignored after reducing platform velocity, increasing capture rate, or using a stop-scan-go motion tactic. A few notable algorithms adapt the traditional discrete-time SLAM formulation to a fundamental form of continuous-time SLAM, by applying frame-to-frame pose-interpolation techniques between keyframes. One such work, by Hedborg et al. (2012), demonstrates the use of inexpensive rolling-shutter cameras to perform full nonlinear bundle adjustment. Using appearance-based lidar data in an identical fashion to this research, Dong and Barfoot (2012) perform frame-to-frame motion-compensated visual odometry. Finally, using dense range data from a 
continuously-spinning 2D lidar, Bosse and Zlot (2009) have developed a technique, akin to the iterative closest point (ICP) algorithm, that is capable of iteratively correcting point clouds for motion distortion. Adaptations of their work using surfels have been successful in mapping a large underground mine (Zlot and Bosse, 2012) and estimating the irregular motion of a 2D lidar attached to the end of a spring (Bosse et al., 2012).

Furgale et al. (2012) formally derive the general continuous-time SLAM problem and demonstrate the use of temporal basis functions to find a parametric solution for a typical camera-IMU calibration problem. The use of uniformly spaced cubic B-splines to parameterize the robot trajectory can also be seen in the estimation schemes derived by Bibby and Reid (2010), and Fleps et al. (2011). Temporal basis functions for continuous-time estimation are also used in the works by Oth et al. (2013) and Anderson et al. (2014), which investigate how parameterizations can be chosen adaptively to prevent underfitting and overfitting the measurements. Lovegrove et al. (2013) formulate a methodology for visual-inertial fusion very similar to the work by Furgale et al. (2012), but parameterize rotations using the cumulative B-spline representation introduced in the computer animation field by Kim et al. (1995). Although the connection was not drawn, their method shares similar properties to our previously published work on the relative continuous-time formulation (Anderson and Barfoot, 2013b); both systems are singularity-free, and perform local estimations that are related to the velocity domain. Finally, in an interesting fusion of state parameterizations, the most recent adaptation of the mining work by Zlot and Bosse (2014) uses low-frequency cubic B-spline perturbations to update a high-frequency set of discrete pose variables. The approach presented in this paper adopts the parametric state representation due to practicality and effectiveness; however, it is worth noting that non-parametric representations, using Gaussian processes, have also been successfully applied in an effort to achieve greater representational power (Tong et al., 2013; Barfoot et al., 2014).

With respect to outlier rejection, the RANSAC algorithm (Fischler and Bolles, 1981) has been highly successful as an outlierrejection scheme in the visual pipeline and has become popularized due to its speed and efficiency. Using global shutter cameras, both the monocular 5-point algorithm (Nistér, 2004) and stereo-pair, 3-point algorithm (Haralick et al., 1994) are widely used, most notably on the Mars Exploration Rovers (Maimone et al., 2007). Although the use of CMOS technology is desirable due to low cost and widespread availability, the addition of a temporal parameter to the standard camera model causes nontrivial and unhandled complexities in the mature visual pipeline. Much of the existing rolling-shutter literature makes use of special cases and there exists only a few pieces of work that properly account for the 6D sensor motion. However, many of these methods stray from using RANSAC as an outlier-rejection scheme.

Early work by Ait-Aider et al. (2006) derives a nonlinear, least-squares estimator for the velocity of a monocular rollingshutter camera over a single frame of data; in order to find this velocity over only a single image, a known geometric target is used, and the feature correspondence is supervised. In a separate work, Ait-Aider and Berry (2009) also derive the batch nonlinear optimization technique for a stereo rig that includes only one rolling-shutter camera, and one global-shutter camera. Again, this experiment uses a target with known geometry and easy-to-find feature correspondences. The possibility of using RANSAC is mentioned, but left as a future extension.

Jia and Evans (2012) apply the extended Kalman filter (EKF) to a monocular rolling-shutter camera and use predictive measurements from a gyroscope and accelerometer. In a similar fashion to RANSAC, this algorithm handles outlier rejection by applying the EKF correction step for multiple feature correspondence hypotheses and checking to see which update generated the most likely set of inliers. Akin to the classic RANSAC algorithm, our proposed method does not require additional sensors, such as a gyroscope, to find feature correspondences.

The primary outlier-rejection scheme for the aforementioned visual pipeline by Hedborg et al. (2012) is a cross-check method, which makes no use of camera geometry and relies on the use of a feature tracker, such as Kanade-Lucas-Tomasi (KLT) (Lucas and Kanade, 1981). After an initial bundle-adjustment step, a second stage of outlier rejection is applied by checking point triangulations from multiple viewpoints. In an earlier work, Hedborg et al. (2011) note that the use of a cross check with KLT is ideal for sensors with a very high framerate. In contrast to the $30 \mathrm{~Hz}$ camera used by Hedborg et al. (2011), the proposed research aims to find feature correspondences between rolling-shutter-type images taken at 2Hz. Furthermore, Hedborg et al. (2011) take advantage of a secondary rejection step that is tightly coupled with the estimation phase of the visual pipeline. In the frame-to-frame estimation technique proposed by Dong and Barfoot (2012), a typical 3-point RANSAC algorithm is applied with a very loose threshold that allows for some outliers. In the estimation phase, a robust M-estimation scheme is then used to try to minimize the effect of incorrectly matched features.

In a traditional fashion, our proposed adaptation of RANSAC is able to perform outlier rejection as a precursor to batch nonlinear estimation. Transitioning away from a rigid transformation model, and instead using the proposed constant-velocity model, our algorithm is able to compensate for motion-distorted data. 


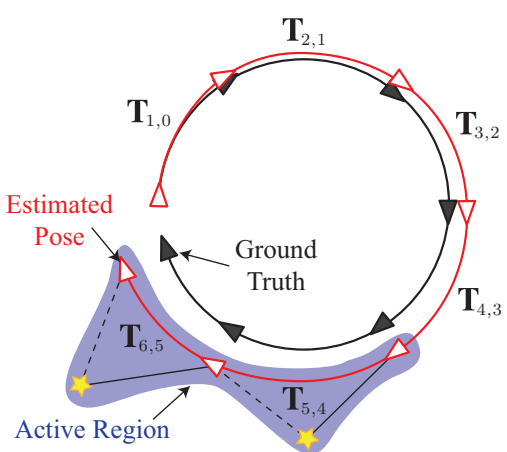

(a) Sliding Window Filter

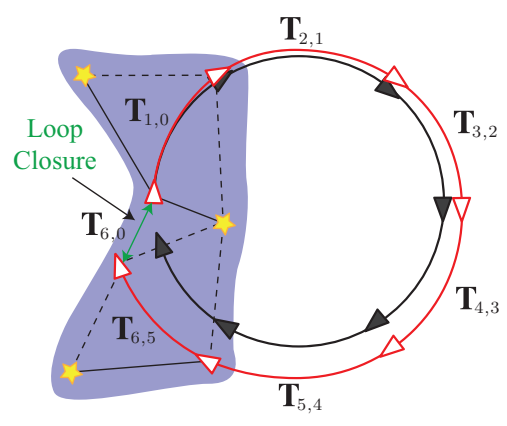

(b) Loop-Closure Optimization

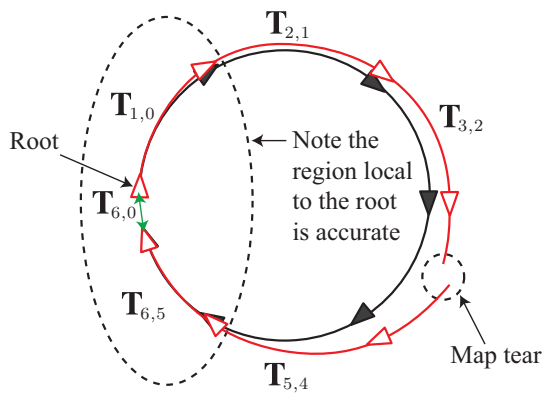

(c) Map Consistency

Figure 6: In the relative SLAM problem formulation (Sibley et al., 2009), the full solution is found incrementally by performing many small batch optimizations. Each of these batch optimization problems estimate a subset of the full state, defined as the active state, consisting of a connected set of poses and the landmarks upon which they depend. (a) In the general open-loop case, the active state is similar to that of a sliding-window-style filter, and usually spans a few of the most recent poses. (b) In the case of a loop closure, the active state contains a few poses from each side; when a landmark is observed from poses on both sides of the loop closure, it contributes information to not only to the loop-closure estimate, but also the local trajectory estimates on each side of the loop closure. (c) The relative formulation leads to a map that is locally accurate, and can be computed in a fast and incremental fashion. However, the map is globally inconsistent, as plotting it in a single privileged coordinate frame, or root frame, causes map tears at the far edges.

In contrast to the majority of continuous-time estimation schemes that are derived in a single privileged coordinate frame, and by extension cannot handle large-scale loop closures in constant time, the estimator presented in this paper adopts the completely relative problem formulation by Sibley et al. (2009). As the proposed relative method is also a continuous-time solution, it maintains the ability to overcome the state-size tractability issues associated with high-rate and scanning sensors by using the parametric framework derived by Furgale et al. (2012). To attain this fusion of techniques, the open-loop trajectory is represented by a continuous-time estimate of the robot's velocity, while large-scale loop closures are described by discrete transformations that relate the robot's pose at two different times.

\section{Relative Coordinates for Continuous-Time Batch Estimation}

In this section, we describe and formulate the relative, continuous-time SLAM problem. Similarly to the typical visual SLAM problem, our goal is to find the optimal solution for the robot trajectory and the map parameters. Although arguably not as simple as the single-privileged coordinate frame, the advantage in using a relative formulation of SLAM is that a window-style filter can still be used to incrementally solve the problem in constant-time, even at loop closure (Sibley et al., 2009). A side benefit is that when the trajectory is parameterized as a velocity profile, it is free of singularities.

\subsection{Relative Coordinates}

Illustrated in Figure 6(a) is the typical sliding-window-style estimation for a bundle-adjustment problem. Each time a new frame is added to the system, an optimization problem is run using a subset of the most recent data to estimate the local pose and landmark parameters in the active state (highlighted in blue). Although the problem has a cubic solve time in the number of poses being optimized, estimating only a local subset of recent poses keeps this computation time constant as the estimator progresses. The advantage of using a relative system is that when a loop closure is observed, the estimator does not need to optimize over all the poses in the trajectory in order to optimize the spatial link and refine the landmark parameters using the additional information; as illustrated in Figure 6(b), a window-style optimization can be performed using temporally linked poses from both places, and the spatial transformation that relates them.

The difference with the relative formulation is that the map is no longer globally consistent, as seen in Figure 6(c). Starting with a root node, the map can be put into a single coordinate frame by compounding poses in a breadth first search (BFS) fashion; this causes map tears at the far edges of loops, as the solution is over-parameterized and therefore multiple pose chains exist between any one pose and another. With the exception of a few select tasks, such as globally optimal path planning, the 
majority of mobile robotic tasks, such as graph-based planning, navigation, or object avoidance, require only locally accurate information and therefore are well-suited to run with a constant-time relative SLAM formulation.

\subsection{Relative Continuous-Time Trajectory Formulation}

In order to combine the relative and continuous-time SLAM formulations, it is proposed that the trajectory be represented by the robot velocity, expressed in the local robot coordinate frame. The intuition behind this decision derives from the nature of both the continuous-time and relative formulations. Consider a system in which we sample the robot's pose at several discrete times and then construct the series of relative pose changes that make up that trajectory. Now, if we consider reducing the sampling period to an infinitesimal level, we note that the series of infinitesimal relative pose change is in essence the robotoriented velocity. Recovering a relative pose change between any two times is simply a matter of integrating the velocity information between them.

\subsubsection{Velocity Profile}

To define the velocity profile, we begin by introducing the coordinate frames $\underline{\mathcal{F}}_{i}$, our inertial reference frame, and $\underline{\mathcal{F}}_{s}(t)$, the robot's instantaneous sensor frame. In the relative, continuous-time formulation of the problem, our algorithm aims to estimate the $6 \mathrm{D}$ velocity of the robot,

$$
\varpi(t):=\left[\begin{array}{c}
\boldsymbol{\nu}_{t}^{t, i} \\
\boldsymbol{\omega}_{t}^{t, i}
\end{array}\right],
$$

which is expressed in the robot's sensor frame over the time interval $t=[0, T]$, where $\nu_{t}^{t, i}$ and $\boldsymbol{\omega}_{t}^{t, i}$ are the linear and angular components. The notation, $\mathbf{v}_{c}^{b, a}$, refers to a vector from $a$ to $b$, expressed in frame $c$. Note that for simplicity, all references to the time-varying sensor frame, $\underset{\rightarrow}{\mathcal{F}}(t)$, have been replaced with the timestamp that we wish to reference, for example, time $t$ in the velocity, $\boldsymbol{\nu}_{t}^{t, i}$. Notably, an advantage of representing the trajectory of the robot as a velocity profile, in contrast to using rotations or transformations, is that there are no constraints that need to be enforced on the parameters of the solution.

\subsubsection{Kinematics}

In order to include measurements that depend on the pose, or pose change, of the robot, in a batch estimation scheme, we must first derive the relationship between the robot velocity, $\varpi(t)$, and the robot pose, $\mathbf{T}(t)$, at time $t$.

We begin with our transformation matrix definition,

$$
\mathbf{T}(t):=\mathbf{T}_{t, i}=\left[\begin{array}{cc}
\mathbf{C}_{t, i} & \mathbf{r}_{t}^{i, t} \\
\mathbf{0}^{T} & 1
\end{array}\right] \equiv\left[\begin{array}{cc}
\mathbf{C}_{t, i} & -\mathbf{C}_{t, i} \mathbf{r}_{i}^{t, i} \\
\mathbf{0}^{T} & 1
\end{array}\right]
$$

where $\mathbf{C}_{t, i}$ is a $3 \times 3$ matrix describing the rotation of the sensor frame with respect to the inertial frame and $\mathbf{r}_{i}^{t, i}$ is the $3 \times 1$ translation of the sensor frame with respect to the inertial frame, expressed in the inertial frame.

The kinematic equation relating angular velocity to rotation is

$$
\dot{\mathbf{C}}_{t, i}=\omega_{t}^{t, i} \mathbf{C}_{t, i}
$$

where $(\cdot)^{\wedge}$ is the skew-symmetric operator (Murray et al., 1994):

$$
\left[\begin{array}{l}
u_{1} \\
u_{2} \\
u_{3}
\end{array}\right]^{\wedge}:=\left[\begin{array}{ccc}
0 & -u_{3} & u_{2} \\
u_{3} & 0 & -u_{1} \\
-u_{2} & u_{1} & 0
\end{array}\right] .
$$


Expanding the time derivative of the translational component, $\dot{\mathbf{r}}_{t}^{i, t}$, we find that

$$
\begin{aligned}
\dot{\mathbf{r}}_{t}^{i, t} & =-\frac{d}{d t}\left(\mathbf{C}_{t, i} \mathbf{r}_{i}^{t, i}\right) \\
& =-\dot{\mathbf{C}}_{t, i} \mathbf{r}_{i}^{t, i}-\mathbf{C}_{t, i} \dot{\mathbf{r}}_{i}^{t, i} \\
& =-\boldsymbol{\omega}_{t}^{t, i} \underbrace{\mathbf{C}_{t, i}\left(-\mathbf{r}_{i}^{i, t}\right)}_{-\mathbf{r}_{t}^{i, t}} \underbrace{-\mathbf{C}_{t, i} \dot{\mathbf{r}}_{i}^{t, i}}_{=: \boldsymbol{\nu}_{t}^{t, i}} \\
& =\boldsymbol{\omega}_{t}^{t, i} \wedge \mathbf{r}_{t}^{i, t}+\boldsymbol{\nu}_{t}^{t, i} .
\end{aligned}
$$

Substituting our kinematic equations, (3) and (4), into the time derivative of the transformation matrix,

$$
\begin{aligned}
\dot{\mathbf{T}}_{t, i} & =\left[\begin{array}{cc}
\dot{\mathbf{C}}_{t, i} & \dot{\mathbf{r}}_{t}^{i, t} \\
\mathbf{0}^{T} & 0
\end{array}\right] \\
& =\left[\begin{array}{cc}
\boldsymbol{\omega}_{t}^{t, i} \mathbf{C}_{t, i} & \boldsymbol{\omega}_{t}^{t, i} \mathbf{r}_{t}^{i, t}+\boldsymbol{\nu}_{t}^{t, i} \\
\mathbf{0}^{T} & 0
\end{array}\right] \\
& =\left[\begin{array}{cc}
\boldsymbol{\omega}_{t}^{t, i} & \boldsymbol{\nu}_{t}^{t, i} \\
\mathbf{0}^{T} & 0
\end{array}\right]\left[\begin{array}{cc}
\mathbf{C}_{t, i} & \mathbf{r}_{t}^{i, t} \\
\mathbf{0}^{T} & 1
\end{array}\right] \\
& =\left[\begin{array}{c}
\boldsymbol{\nu}_{t}^{t, i} \\
\boldsymbol{\omega}_{t}^{t, i}
\end{array}\right]^{\wedge} \mathbf{T}_{t, i}, \\
\dot{\mathbf{T}}_{t, i} & =\varpi(t)^{\wedge} \mathbf{T}_{t, i},
\end{aligned}
$$

where $(\cdot)^{\wedge}$ is the overloaded SE(3) operator (Murray et al., 1994; Barfoot and Furgale, 2014),

$$
\mathbf{w}^{\wedge}=\left[\begin{array}{l}
\mathbf{u} \\
\mathbf{v}
\end{array}\right]^{\wedge}:=\left[\begin{array}{ll}
\mathbf{v}^{\wedge} & \mathbf{u} \\
\mathbf{0}^{T} & 0
\end{array}\right]
$$

Integrating (5) gives us the desired relationship between $\mathbf{T}_{t, i}$ and the robot velocity, $\varpi(t)$,

$$
\mathbf{T}_{t, i}=\mathbf{T}_{0, i}+\int_{0}^{t} \varpi(\tau)^{\wedge} \mathbf{T}_{\tau, i} d \tau,
$$

where $\mathbf{T}_{0, i}$ is the initial robot pose, at time zero. As we are mostly interested in the pose change of the robot between two non-zero times, $t_{a}$ and $t_{b}$, we aim to find a general expression for the matrix $\mathbf{T}_{t_{b}, t_{a}}$. In order to replace the inertial reference frame, ${\underset{\mathcal{F}}{\rightarrow}}_{i}$, in (5) with the sensor frame at time $t_{a}, \underset{\mathcal{F}}{\rightarrow}\left(t_{a}\right)$, we begin with the expression,

$$
\mathbf{T}_{t, i}=\mathbf{T}_{t, t_{a}} \mathbf{T}_{t_{a}, i}
$$

and take the derivative,

$$
\dot{\mathbf{T}}_{t, i}=\dot{\mathbf{T}}_{t, t_{a}} \mathbf{T}_{t_{a}, i}+\underbrace{\mathbf{T}_{t, t_{a}} \dot{\mathbf{T}}_{t_{a}, i}}_{\text {zero }}=\dot{\mathbf{T}}_{t, t_{a}} \mathbf{T}_{t_{a}, i}
$$

noting that $\mathbf{T}_{t, t_{a}} \dot{\mathbf{T}}_{t_{a}, i}$ is zero due to the fact that $\mathbf{T}_{t_{a}, i}$ does not depend on $t$. By manipulating (5) and (9), we find that

$$
\dot{\mathbf{T}}_{t, t_{a}}=\varpi(t)^{\wedge} \mathbf{T}_{t, t_{a}}
$$

The time integral over the period $t_{a}$ to $t_{b}$ then gives us

$$
\mathbf{T}_{t_{b}, t_{a}}=\mathbf{1}+\int_{t_{a}}^{t_{b}} \varpi(t)^{\wedge} \mathbf{T}_{t, t_{a}} d t
$$


where 1 is the identity matrix. Note that the integrations in (7) and (11) need to be calculated using a method that preserves the properties of the transformation matrix. One way to do this is using the exponential map (Barfoot and Furgale, 2014),

$$
\begin{aligned}
\mathbf{T} & :=\exp \left(\boldsymbol{\xi}^{\wedge}\right)=\left[\begin{array}{cc}
\mathbf{C}(\phi) & \mathbf{J}(\phi) \boldsymbol{\rho} \\
\mathbf{0}^{T} & 1
\end{array}\right], \quad \boldsymbol{\xi}:=\left[\begin{array}{l}
\boldsymbol{\rho} \\
\phi
\end{array}\right], \\
\mathbf{C}(\phi) & :=\cos \phi \mathbf{1}+(1-\cos \phi) \mathbf{a a}^{T}+\sin \phi \mathbf{a}^{\wedge}, \\
\mathbf{J}(\phi) & :=\frac{\sin \phi}{\phi} \mathbf{1}+\left(1-\frac{\sin \phi}{\phi}\right) \mathbf{a}^{T}+\frac{1-\cos \phi}{\phi} \mathbf{a}^{\wedge},
\end{aligned}
$$

where $\rho$ is a translational component and $\phi$ uses the axis-angle parameterization, $\phi:=\phi \mathbf{a}$, such that $\phi$ is the angle and $\mathbf{a}$ is a unit-length vector representing the axis of rotation. For a constant velocity, $\varpi$, we note the relationship $\xi=\Delta t \cdot \varpi$. In implementation, we approximate the solution to (11) numerically by sampling $\varpi(t)$ with a small timestep, $\Delta t$, and creating a chain of transformation matrices that span from time $t_{a}$ to $t_{b}$.

$$
\mathbf{T}_{t_{b}, t_{a}} \approx \underbrace{\exp \left(\Delta t \cdot \varpi\left(t_{b}-\Delta t\right)^{\wedge}\right)}_{\approx \mathbf{T}_{t_{b}, t_{b}-\Delta t}} \cdots \underbrace{\exp \left(\Delta t \cdot \varpi\left(t_{a}+\Delta t\right)^{\wedge}\right)}_{\approx \mathbf{T}_{t_{a}+2 \Delta t, t_{a}+\Delta t}} \underbrace{\exp \left(\Delta t \cdot \varpi\left(t_{a}\right)^{\wedge}\right)}_{\approx \mathbf{T}_{t_{a}+\Delta t, t_{a}}}
$$

Alternatively, we note that for very small pose changes, the exponential map can be approximated using

$$
\exp \left(\boldsymbol{\xi}^{\wedge}\right) \approx \mathbf{1}+\boldsymbol{\xi}^{\wedge} .
$$

Determining an appropriate integration timestep, $\Delta t$, is subject to the required accuracy of the integration, the typical length of an integration (in time), and the typical magnitude of $\varpi(t)$; in practice we use a maximum step value of $\Delta t=0.01$, with smaller steps when we wish to extract the pose at specific intermediate times (e.g., you may want the pose change at several measurement times, spaced less than 0.01 seconds apart).

The integral, presented in (11) and approximated in (13), relates the continuous-time velocity profile, $\varpi(t)$, to a discrete pose change, $\mathbf{T}_{t_{b}, t_{a}}$, and is an important result, as it allow us to calculate the value of expected measurements that depend on pose (e.g., visual measurements of an estimated landmark) in our relative continuous-time formulation.

\subsubsection{Perturbations to the Kinematics}

In order to linearize terms involving a transform integrated from the velocity profile, using (11), we extend our discussion of kinematics to consider the effect of small changes to $\varpi(t)$. Using the perturbation scheme,

$$
\mathbf{T}=\exp \left(\boldsymbol{\xi}^{\wedge}\right)=\exp \left(\delta \boldsymbol{\xi}^{\wedge}\right) \exp \left(\overline{\boldsymbol{\xi}}^{\wedge}\right) \approx\left(\mathbf{1}+\delta \boldsymbol{\xi}^{\wedge}\right) \overline{\mathbf{T}},
$$

the kinematic equation, $\dot{\mathbf{T}}=\varpi^{\wedge} \mathbf{T}$, becomes

$$
\frac{d}{d t}\left(\left(\mathbf{1}+\delta \boldsymbol{\xi}^{\wedge}\right) \overline{\mathbf{T}}\right) \approx(\bar{\varpi}+\delta \varpi)^{\wedge}\left(\mathbf{1}+\delta \boldsymbol{\xi}^{\wedge}\right) \overline{\mathbf{T}} .
$$

By expanding and dropping small terms, we find

$$
\dot{\overline{\mathbf{T}}}+\delta \boldsymbol{\xi}^{\wedge} \dot{\overline{\mathbf{T}}}+\delta \dot{\boldsymbol{\xi}}^{\wedge} \overline{\mathbf{T}} \approx \bar{\varpi}^{\wedge} \overline{\mathbf{T}}+\bar{\varpi}^{\wedge} \delta \boldsymbol{\xi}^{\wedge} \overline{\mathbf{T}}+\delta \varpi^{\wedge} \overline{\mathbf{T}} .
$$

Using the nominal solution, $\dot{\overline{\mathbf{T}}}=\bar{\varpi}^{\wedge} \overline{\mathbf{T}}$, and the identity, $\boldsymbol{\xi}_{1}^{\wedge} \boldsymbol{\xi}_{2}^{\wedge}-\boldsymbol{\xi}_{2}^{\wedge} \boldsymbol{\xi}_{1}^{\wedge} \equiv\left(\boldsymbol{\xi}_{1}^{\curlywedge} \boldsymbol{\xi}_{2}\right)^{\wedge}$, where $(\cdot)^{\curlywedge}$ is the SE(3) operator (Barfoot and Furgale, 2014),

$$
\mathbf{w}^{\curlywedge}=\left[\begin{array}{l}
\mathbf{u} \\
\mathbf{v}
\end{array}\right]^{\curlywedge}:=\left[\begin{array}{ll}
\mathbf{v}^{\wedge} & \mathbf{u}^{\wedge} \\
\mathbf{0} & \mathbf{v}^{\wedge}
\end{array}\right],
$$

equation (17) can be manipulated to find,

$$
\begin{aligned}
\delta \dot{\boldsymbol{\xi}}^{\wedge} \bar{\varpi}^{\wedge} \overline{\mathbf{T}}+\delta \dot{\boldsymbol{\xi}}^{\wedge} \overline{\mathbf{T}} & \approx \bar{\varpi}^{\wedge} \delta \boldsymbol{\xi}^{\wedge} \overline{\mathbf{T}}+\delta \varpi^{\wedge} \overline{\mathbf{T}} \\
\delta \dot{\boldsymbol{\xi}}^{\wedge} & \approx-\delta \boldsymbol{\xi}^{\wedge} \bar{\varpi}^{\wedge}+\bar{\varpi}^{\wedge} \delta \boldsymbol{\xi}^{\wedge}+\delta \varpi^{\wedge} \\
& =\left(\bar{\varpi}^{\curlywedge} \delta \boldsymbol{\xi}\right)^{\wedge}+\delta \varpi^{\wedge} \\
\delta \dot{\boldsymbol{\xi}} & \approx \bar{\varpi}^{\curlywedge} \delta \boldsymbol{\xi}+\delta \varpi .
\end{aligned}
$$


Giving us the nominal and perturbed equations:

$$
\begin{aligned}
\dot{\overline{\mathbf{T}}}(t) & =\bar{\varpi}(t)^{\wedge} \overline{\mathbf{T}}(t), \\
\delta \dot{\boldsymbol{\xi}}(t) & =\bar{\varpi}(t)^{\curlywedge} \delta \boldsymbol{\xi}(t)+\delta \varpi(t),
\end{aligned}
$$

which can be integrated separately and combined to provide the full solutions. We have shown in (13) how the nonlinear nominal solution can be integrated numerically. The perturbed equation is a linear time-varying equation of the form

$$
\dot{\mathbf{x}}(t)=\mathbf{A}(t) \mathbf{x}(t)+\mathbf{B}(t) \mathbf{u}(t) .
$$

The general solution to the initial value problem is given by

$$
\mathbf{x}(t)=\mathbf{\Phi}(t, 0) \mathbf{x}(0)+\int_{0}^{t} \mathbf{\Phi}(t, \tau) \mathbf{B}(\tau) \mathbf{u}(\tau) d \tau
$$

where $\boldsymbol{\Phi}(t, \tau)$ is called the state transition matrix and satisfies

$$
\begin{aligned}
\dot{\boldsymbol{\Phi}}(t, \tau) & =\mathbf{A}(t) \boldsymbol{\Phi}(t, \tau), \\
\mathbf{\Phi}(\tau, \tau) & =\mathbf{1} .
\end{aligned}
$$

The state transition matrix always exists and is unique, but it cannot always be found analytically. For our particular perturbed equation, it can be expressed as:

$$
\boldsymbol{\Phi}(t, \tau)=\overline{\mathcal{T}}(t) \overline{\mathcal{T}}(\tau)^{-1} .
$$

where $\mathcal{T}$ is the adjoint transformation matrix (Barfoot and Furgale, 2014), given by

$$
\mathcal{T}:=\operatorname{Ad}(\mathbf{T})=\operatorname{Ad}\left(\left[\begin{array}{cc}
\mathbf{C} & \mathbf{r} \\
\mathbf{0}^{T} & 1
\end{array}\right]\right)=\left[\begin{array}{cc}
\mathbf{C} & \mathbf{r}^{\wedge} \mathbf{C} \\
\mathbf{0} & \mathbf{C}
\end{array}\right]=\exp \left(\boldsymbol{\xi}^{\curlywedge}\right) .
$$

To verify, we check that (24) satisfies the required conditions of a state transition matrix:

$$
\begin{aligned}
& \dot{\boldsymbol{\Phi}}(t, \tau)=\frac{d}{d t}\left(\overline{\mathcal{T}}(t) \overline{\mathcal{T}}(\tau)^{-1}\right)=\dot{\mathcal{\mathcal { T }}}(t) \overline{\mathcal{T}}(\tau)^{-1}=\bar{\varpi}(t)^{\curlywedge} \overline{\mathcal{T}}(t) \overline{\mathcal{T}}(\tau)^{-1}=\bar{\varpi}(t)^{\curlywedge} \mathbf{\Phi}(t, \tau), \\
& \mathbf{\Phi}(\tau, \tau)=\overline{\mathcal{T}}(\tau) \overline{\mathcal{T}}(\tau)^{-1}=\mathbf{1}
\end{aligned}
$$

Using the general solution to the initial value problem, we have that:

$$
\delta \boldsymbol{\xi}(t)=\overline{\mathcal{T}}(t) \overline{\mathcal{T}}(0)^{-1} \delta \boldsymbol{\xi}(0)+\overline{\mathcal{T}}(t) \int_{0}^{t} \overline{\mathcal{T}}(\tau)^{-1} \delta \varpi(\tau) d \tau
$$

To verify again that this is indeed the correct solution, we can differentiate:

$$
\begin{aligned}
\delta \dot{\boldsymbol{\xi}}(t) & =\dot{\overline{\mathcal{T}}}(t) \overline{\mathcal{T}}(0)^{-1} \delta \boldsymbol{\xi}(0)+\dot{\mathcal{\mathcal { T }}}(t) \int_{0}^{t} \overline{\mathcal{T}}(\tau)^{-1} \delta \varpi(\tau) d \tau+\overline{\mathcal{T}}(t) \overline{\mathcal{T}}(t)^{-1} \delta \varpi(t) \\
& =\bar{\varpi}(t)^{\curlywedge} \underbrace{\left(\overline{\mathcal{T}}(t) \overline{\mathcal{T}}(0)^{-1} \delta \boldsymbol{\xi}(0)+\overline{\mathcal{T}}(t) \int_{0}^{t} \overline{\mathcal{T}}(\tau)^{-1} \delta \varpi(\tau) d \tau\right)}_{\delta \boldsymbol{\xi}(t)}+\delta \varpi(t) \\
& =\bar{\varpi}(t)^{\curlywedge} \delta \boldsymbol{\xi}(t)+\delta \varpi(t) .
\end{aligned}
$$

Similar to the extension of (7) to (11), we wish to consider the extension of (27) a general pose change, $\mathbf{T}_{t_{b}, t_{a}}$, between two non-zero times, $t_{a}$ and $t_{b}$. Using the usual perturbation scheme, and the identity (Barfoot and Furgale, 2014),

$$
\mathbf{T} \exp \left(\mathbf{w}^{\wedge}\right) \equiv \exp \left((\mathcal{T} \mathbf{w})^{\wedge}\right) \mathbf{T}
$$


it is straightforward to derive the relationship (Tong et al., 2014),

$$
\mathbf{T}_{t_{b}, t_{a}} \approx\left(\mathbf{1}+\delta \boldsymbol{\xi}_{t_{b}, t_{a}}^{\wedge}\right) \overline{\mathbf{T}}_{t_{b}, t_{a}} \approx\left(\mathbf{1}+\left(\delta \boldsymbol{\xi}\left(t_{b}\right)-\overline{\mathcal{T}}\left(t_{b}\right) \overline{\mathcal{T}}\left(t_{a}\right)^{-1} \delta \boldsymbol{\xi}\left(t_{a}\right)\right)^{\wedge}\right) \overline{\mathbf{T}}_{t_{b}, t_{a}}
$$

where,

$$
\delta \boldsymbol{\xi}_{t_{b}, t_{a}} \approx \delta \boldsymbol{\xi}\left(t_{b}\right)-\overline{\mathcal{T}}\left(t_{b}\right) \overline{\mathcal{T}}\left(t_{a}\right)^{-1} \delta \boldsymbol{\xi}\left(t_{a}\right) .
$$

To simplify the term $\delta \boldsymbol{\xi}\left(t_{b}\right)-\overline{\mathcal{T}}\left(t_{b}\right) \overline{\mathcal{T}}\left(t_{a}\right)^{-1} \delta \boldsymbol{\xi}\left(t_{a}\right)$, and relate the perturbation back to our velocity profile, $\varpi(t)$, we substitute the solution for $\delta \boldsymbol{\xi}(t)$ found in (27):

$$
\begin{aligned}
\delta \boldsymbol{\xi}\left(t_{b}\right)-\overline{\mathcal{T}}\left(t_{b}\right) \overline{\mathcal{T}}\left(t_{a}\right)^{-1} \delta \boldsymbol{\xi}\left(t_{a}\right)= & \left(\overline{\mathcal{T}}\left(t_{b}\right) \overline{\mathcal{T}}(0)^{-1} \delta \boldsymbol{\xi}(0)+\overline{\mathcal{T}}\left(t_{b}\right) \int_{0}^{t_{b}} \overline{\mathcal{T}}(\tau)^{-1} \delta \varpi(\tau) d \tau\right) \\
& -\overline{\mathcal{T}}\left(t_{b}\right) \overline{\mathcal{T}}\left(t_{a}\right)^{-1}\left(\overline{\mathcal{T}}\left(t_{a}\right) \overline{\mathcal{T}}(0)^{-1} \delta \boldsymbol{\xi}(0)+\overline{\mathcal{T}}\left(t_{a}\right) \int_{0}^{t_{a}} \overline{\mathcal{T}}(\tau)^{-1} \delta \varpi(\tau) d \tau\right) \\
= & \overline{\mathcal{T}}\left(t_{b}\right)\left(\int_{0}^{t_{b}} \overline{\mathcal{T}}(\tau)^{-1} \delta \varpi(\tau) d \tau-\int_{0}^{t_{a}} \overline{\mathcal{T}}(\tau)^{-1} \delta \varpi(\tau) d \tau\right) \\
= & \int_{t_{a}}^{t_{b}} \overline{\mathcal{T}}_{t_{b}, \tau} \delta \varpi(\tau) d \tau .
\end{aligned}
$$

Substituting (32) back into (30), we find the following relationship between $\mathbf{T}_{t_{b}, t_{a}}$ and small changes in $\varpi(t)$,

$$
\mathbf{T}_{t_{b}, t_{a}} \approx\left(\mathbf{1}+\left(\int_{t_{a}}^{t_{b}} \overline{\mathcal{T}}_{t_{b}, \tau} \delta \varpi(\tau) d \tau\right)^{\wedge}\right) \overline{\mathbf{T}}_{t_{b}, t_{a}} .
$$

Although it is initially unclear how one might isolate the time-varying parameter, $\delta \varpi(t)$, since it is embedded in the integral, the following section will show how this is made manageable with the use of parametric basis functions.

The relationship in (33) between a pose change, $\mathbf{T}_{t_{b}, t_{a}}$, and a perturbation to the velocity profile, $\delta \varpi(t)$, allows us to use this relative continuous-time formulation with the gradient-based optimization technique described in the next section. The nominal pose change, $\overline{\mathbf{T}}_{t_{b}, t_{a}}$, is integrated from the nominal velocity profile, $\bar{\varpi}(t)$, using (11).

\subsection{Parametric Batch Estimator}

\subsubsection{Trajectory}

In the relative, continuous-time formulation of the SLAM problem, we wish to estimate the 6D velocity of the robot, $\varpi(t)$, which is expressed in the robot's sensor frame, over the time interval $t=[0, T]$. Moving forward in the derivation of the estimator, we define a parametric representation for the continuous-time state. We choose to do this by using a weighted sum of temporal basis functions,

$$
\varpi(t):=\Psi(t) \mathbf{c}, \quad \boldsymbol{\Psi}(t):=\left[\begin{array}{llll}
\boldsymbol{\psi}_{1}(t) & \boldsymbol{\psi}_{2}(t) & \cdots & \boldsymbol{\psi}_{6 B}(t)
\end{array}\right], \quad \mathbf{c}:=\left[\begin{array}{llll}
c_{1} & c_{2} & \cdots & c_{6 B}
\end{array}\right]^{T},
$$

where we have $B$ temporal basis functions in each of the six problem dimensions, and each basis function, $\boldsymbol{\psi}_{b}(t)$, is a $6 \times 1$ column vector. The basis functions' coefficients, $\mathbf{c}$, are the free parameters that can be used to vary the velocity estimate, $\varpi(t)$; using a parametric representation, the estimation goal is to simply find the optimal values of these weights.

Due to the requirement that our basis functions have local support, which will be discussed later, our implementation uses cubic B-spline basis functions, as seen in Figure 7. An important result of the parametric representation is that the timevarying component, $\boldsymbol{\Psi}(t)$, is separated from the variables that we wish to estimate, $\mathbf{c}$; this separability is necessary in order to formulate a typical nonlinear optimization problem with a set of discrete, time-invariant state variables. Furthermore, as we will see in Section 3.3.6, separating the undetermined component, $\mathbf{c}$, allows us to evaluate the integral in (33).

\subsubsection{Loop Closures}

In addition to the relative trajectory, we also wish to estimate any loop-closure transformations between segments of the trajectory from distinctly different times. These loop closures are stored as $4 \times 4$ homogeneous transformation matrices,

$$
\mathbf{T}_{t_{2}, t_{1}}^{\mathrm{LC}}:=\mathbf{T}\left(t_{2}\right) \mathbf{T}\left(t_{1}\right)^{-1}
$$




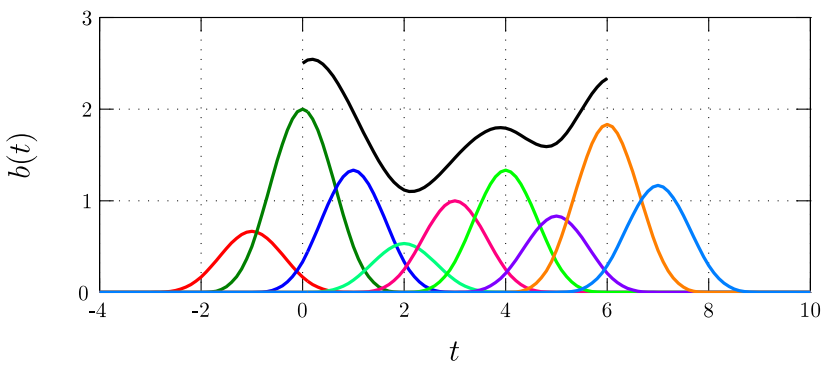

Figure 7: This figure illustrates our use of cubic B-spline basis functions in one dimension. A single cubic B-spline is a smooth piecewise polynomial defined over four time segments. The points in time that separate two time segments are known as knots. We use a uniform knot spacing, such that only four cubic B-splines are nonzero at any time. The example shown here has nine uniformly spaced cubic B-splines that are used to define the weighted sum (black) over six time segments.

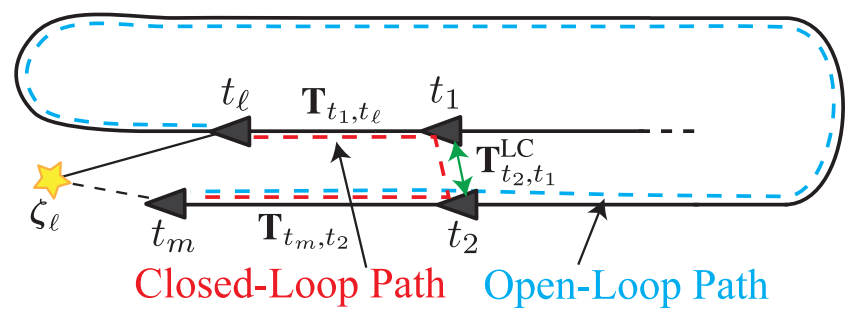

Figure 8: In order to formulate an expected measurement, $\hat{\mathbf{y}}_{\ell, m}$, the point $\boldsymbol{\zeta}_{\ell}$, which is expressed in the sensor frame at time $t_{\ell}$, needs to be transformed to the current observation time, $t_{m}$. In the general open-loop case (seen in blue), the transform, $\mathbf{T}_{t_{m}, t_{\ell}}$, is found by simply integrating the continuous-time velocity profile. However, the existence of loop closures create alternate integration paths (seen in red). Integration of the shortest temporal path is often the most desirable, as it incurs the least estimation error.

between the sensor frame, ${\underset{\mathcal{F}}{s}}_{s}(t)$, at the two distinct times, $t_{1}$ and $t_{2}$. As described in Section 3.1, these loop-closure transformations over parameterize the trajectory solution provided by $\varpi(t)$, but allow us to perform loop-closure optimizations using a window-style filter; these optimizations are constant time and generate locally accurate maps.

\subsubsection{Landmarks}

In addition to estimating the robot velocity and loop closures, we wish to estimate the 3D position of the landmark parameters,

$$
\zeta=\left[\begin{array}{c}
\boldsymbol{\zeta}_{1} \\
\vdots \\
\zeta_{L}
\end{array}\right], \quad \boldsymbol{\zeta}_{\ell}=\mathbf{P}_{\ell} \boldsymbol{\zeta}
$$

where $\ell=1 \ldots L, \mathbf{P}_{\ell}$ is a projection matrix that selects the $3 \mathrm{D}$ position $\boldsymbol{\zeta}_{\ell}$ from the state vector $\boldsymbol{\zeta}$, and we have a set of $M$ measurements, $\mathbf{y}_{\ell, m}, m=1 \ldots M$, for each landmark. In order to simplify the notation, we denote $t_{m}:=t_{\ell, m}$, the time of measurement $\mathbf{y}_{\ell, m}$. Similarly, we define $t_{\ell}:=t_{\ell, 1}$, the first measurement time of the $\ell^{\text {th }}$ landmark. Note that in order to use relative coordinates, it is important that each landmark position is stored locally; this is implemented by expressing, $\boldsymbol{\zeta}_{\ell}$, in frame $\mathcal{F}_{s}\left(t_{\ell}\right)$, the sensor pose at the first measurement time. The reason the landmarks must be stored locally is so that the pose change between the frame in which the landmark is stored, and the frames in which it is observed, relies only on temporally local state variables (and not the entire trajectory).

\subsubsection{Optimization Problem}

The joint state that we wish to estimate is defined as $\mathbf{z}:=\{\boldsymbol{\theta}, \boldsymbol{\zeta}\}$, where the state $\boldsymbol{\theta}$ contains parameters related to robot pose, and $\zeta$ is the vector of landmark positions. In the open-loop case, $\boldsymbol{\theta}:=\mathbf{c}$, however, if a loop closure is part of the optimization problem, $\boldsymbol{\theta}:=\left\{\mathbf{c}, \mathbf{T}_{t_{2}, t_{1}}^{\mathrm{LC}}\right\}$.

The objective function that we wish to minimize is

$$
J(\mathbf{z}):=J_{s}(\mathbf{z})+J_{u}(\mathbf{c}), \quad J_{s}(\mathbf{z}):=\frac{1}{2} \sum_{\ell=1}^{L} \sum_{m=1}^{M} \mathbf{e}_{\ell, m}^{T} \mathbf{R}_{\ell, m}^{-1} \mathbf{e}_{\ell, m}, \quad J_{u}(\mathbf{c}):=\frac{1}{2} \int_{t=0}^{T} \mathbf{e}_{u}(\tau)^{T} \mathbf{Q}^{-1} \mathbf{e}_{u}(\tau) d \tau,
$$

where $J_{s}$ is a cost associated with the landmark measurement error, $\mathbf{e}_{\ell, m}$, and $J_{u}$ is a cost associated with motion (i.e., a motion prior). The derivation of $J_{u}$ can be found in Furgale et al. (2012). Furthermore, we note that loop-closure constraints are introduced in the estimation problem solely through the landmarks that are observed from both of the matched trajectory segments, as discussed in the next subsection. 


\subsubsection{Measurements and Costs}

The sensor model for a single landmark measurement is

$$
\mathbf{y}_{\ell, m}:=\mathbf{f}\left(\mathbf{T}_{t_{m}, t_{\ell}} \mathbf{p}_{\ell}\right)+\mathbf{n}_{\ell, m}, \quad \mathbf{p}_{\ell}:=\left[\begin{array}{c}
\boldsymbol{\zeta}_{\ell} \\
1
\end{array}\right],
$$

where $\mathbf{f}(\cdot)$ is the nonlinear camera model, $\mathbf{T}_{t_{m}, t_{\ell}}$ is the $4 \times 4$ homogeneous transform matrix that specifies the pose change in the sensor frame between the measurement times $t_{\ell}$ and $t_{m}, \mathbf{p}_{\ell}$ is the position of landmark $\ell$ in homogeneous coordinates, and the measurement noise, $\mathbf{n}_{\ell, m} \sim \mathcal{N}\left(\mathbf{0}, \mathbf{R}_{\ell, m}\right)$, is assumed to be normally distributed with covariance $\mathbf{R}_{\ell, m}$.

The measurement error is given by,

$$
\mathbf{e}_{\ell, m}=: \mathbf{y}_{\ell, m}-\hat{\mathbf{y}}_{\ell, m}=\mathbf{y}_{\ell, m}-\mathbf{f}\left(\mathbf{T}_{t_{m}, t_{\ell}} \mathbf{p}_{\ell}\right),
$$

where $\hat{\mathbf{y}}_{\ell, m}$ is the expected sensor measurement based on the current state estimate. In the general open-loop case, the unknown transforms, $\mathbf{T}_{t_{m}, t_{\ell}}$, are found by simply integrating over the continuous-time velocity profile. However, in the case that the shortest temporal path between $t_{\ell}$ and $t_{m}$ involves a loop closure, we note that $\mathbf{T}_{t_{m}, t_{\ell}}$ becomes the composition of the following nonlinear transformations,

$$
\mathbf{T}_{t_{m}, t_{\ell}}=\mathbf{T}_{t_{m}, t_{2}} \mathbf{T}_{t_{2}, t_{1}}^{\mathrm{LC}} \mathbf{T}_{t_{1}, t_{\ell}}
$$

where $\mathbf{T}_{t_{m}, t_{2}}$ and $\mathbf{T}_{t_{1}, t_{\ell}}$ are integrated using the velocity profile, and $\mathbf{T}_{\mathrm{LC}}$ exists as a discrete loop-closure transformation between times $t_{1}$ and $t_{2}$. These alternate integration paths are illustrated in Figure 8.

In addition to the typical measurement error cost function, we also wish to add a cost term associated with motion. Assuming no knowledge of the control signal, we model the motion as a zero-mean white noise on acceleration. This can be written as the Gaussian process

$$
\mathbf{e}_{u}(t):=\dot{\varpi}(t)=\mathbf{w}(t), \quad \mathbf{w}(t) \sim \mathcal{G} \mathcal{P}\left(\mathbf{0}, \mathbf{Q} \delta\left(t-t^{\prime}\right)\right),
$$

where $\mathbf{Q}$ is the power-spectral-density matrix and $\delta(\cdot)$ is Dirac's delta function.

\subsubsection{Linearized Cost Terms}

In order to minimize our objective function, $J(\mathbf{z})$, we aim to make it quadratic in a small perturbation of the state, $\delta \mathbf{z}:=$ $\left[\begin{array}{ll}\delta \boldsymbol{\theta}^{T} & \delta \boldsymbol{\zeta}^{T}\end{array}\right]^{T}$, and find the optimal state update $\delta \mathbf{z}^{*}$ by solving $\frac{\partial^{T} J}{\partial \delta \mathbf{z}}=\mathbf{0}$; in the open-loop case, $\delta \boldsymbol{\theta}:=\delta \mathbf{c}$, and in the closedloop case, $\delta \boldsymbol{\theta}:=\left[\begin{array}{ll}\delta \mathbf{c}^{T} & \delta \boldsymbol{\xi}_{t_{2}, t_{1}}^{\mathrm{LC}}\end{array}\right]^{T}$. To solve the system, we must first linearize the error terms with respect to $\delta \mathbf{z}$ and then find an initial guess for the nominal solution, $\overline{\mathbf{z}}$. We note that the linearization of $J_{u}$, is the same as the one shown in Furgale et al. (2012), and therefore this paper will focus on the linearization of $J_{s}$.

Noting that (39) has a composition of two nonlinearities, one for the camera model and one for transforming the landmark into the sensor frame, we begin by defining

$$
\mathbf{g}_{\ell, m}(\mathbf{z}):=\mathbf{T}_{t_{m}, t_{\ell}} \mathbf{p}_{\ell}
$$

From the temporal basis function definition, $\varpi(t)=\boldsymbol{\Psi}(t) \mathbf{c}$, it is clear that $\bar{\varpi}(t)+\delta \varpi(t)=\boldsymbol{\Psi}(t)(\overline{\mathbf{c}}+\delta \mathbf{c})$ and therefore $\delta \varpi(t)=\Psi(t) \delta$ c. Substituting this relationship into (33),

$$
\mathbf{T}_{t_{b}, t_{a}} \approx\left(\mathbf{1}+\left(\int_{t_{a}}^{t_{b}} \overline{\mathcal{T}}_{t_{b}, \tau} \mathbf{\Psi}(\tau) d \tau \delta \mathbf{c}\right)^{\wedge}\right) \overline{\mathbf{T}}_{t_{b}, t_{a}} .
$$

With respect to the landmark position,

$$
\mathbf{p}_{\ell}=\overline{\mathbf{p}}_{\ell}+\mathbf{D} \delta \boldsymbol{\zeta}_{\ell}, \quad \mathbf{D}:=\left[\begin{array}{ccc}
1 & 0 & 0 \\
0 & 1 & 0 \\
0 & 0 & 1 \\
0 & 0 & 0
\end{array}\right] .
$$


Applying these perturbations for an open-loop trajectory, we find that

$$
\begin{aligned}
\mathbf{g}_{\ell, m}(\mathbf{z}) & \approx\left(\mathbf{1}+\left(\int_{t_{\ell}}^{t_{m}} \overline{\mathcal{T}}_{t_{m}, \tau} \boldsymbol{\Psi}(\tau) d \tau \delta \mathbf{c}\right)^{\wedge}\right) \overline{\mathbf{T}}_{t_{m}, t_{\ell}}\left(\overline{\mathbf{p}}_{\ell}+\mathbf{D} \delta \boldsymbol{\zeta}_{\ell}\right) \\
& \approx \overline{\mathbf{T}}_{t_{m}, t_{\ell}} \overline{\mathbf{p}}_{\ell}+\left(\int_{t_{\ell}}^{t_{m}} \overline{\mathcal{T}}_{t_{m}, \tau} \boldsymbol{\Psi}(\tau) d \tau \delta \mathbf{c}\right)^{\wedge} \overline{\mathbf{T}}_{t_{m}, t_{\ell}} \overline{\mathbf{p}}_{\ell}+\overline{\mathbf{T}}_{t_{m}, t_{\ell}} \mathbf{D} \delta \boldsymbol{\zeta}_{\ell} \\
& =\overline{\mathbf{T}}_{t_{m}, t_{\ell}} \overline{\mathbf{p}}_{\ell}+\left[\left(\overline{\mathbf{T}}_{t_{m}, t_{\ell}} \overline{\mathbf{p}}_{\ell} \odot \int_{t_{\ell}}^{t_{m}} \overline{\mathcal{T}}_{t_{m}, \tau} \boldsymbol{\Psi}(\tau) d \tau \quad \overline{\mathbf{T}}_{t_{m}, t_{\ell}} \mathbf{D} \mathbf{P}_{\ell}\right]\left[\begin{array}{l}
\delta \mathbf{c} \\
\delta \boldsymbol{\zeta}
\end{array}\right]\right. \\
& =\overline{\mathbf{g}}_{\ell, m}+\mathbf{G}_{\ell, m} \delta \mathbf{z},
\end{aligned}
$$

correct to first order, where

$$
\begin{aligned}
& \overline{\mathbf{g}}_{\ell, m}:=\overline{\mathbf{T}}_{t_{m}, t_{\ell}} \overline{\mathbf{p}}_{\ell},
\end{aligned}
$$

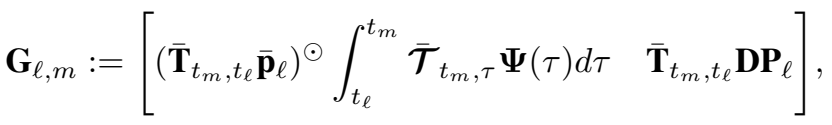

and $(\cdot)^{\odot}$ is the homogeneous coordinate operator (Barfoot and Furgale, 2014),

$$
\mathbf{y}^{\odot}:=\left[\begin{array}{l}
\varepsilon \\
\eta
\end{array}\right]^{\odot}=\left[\begin{array}{cc}
\eta \mathbf{1} & -\varepsilon^{\wedge} \\
\mathbf{0}^{T} & \mathbf{0}^{T}
\end{array}\right]
$$

that is associated with the following useful identities:

$$
\begin{aligned}
\mathbf{w}^{\wedge} \mathbf{y} & \equiv \mathbf{y}^{\odot} \mathbf{w}, \\
(\mathbf{T y})^{\odot} & \equiv \mathbf{T y}^{\odot} \mathcal{T}^{-1} .
\end{aligned}
$$

Returning to our measurement error term,

$$
\begin{aligned}
\mathbf{e}_{\ell, m} & \approx \mathbf{y}_{\ell, m}-\mathbf{f}\left(\overline{\mathbf{g}}_{\ell, m}+\mathbf{G}_{\ell, m} \delta \mathbf{z}\right) \\
& \approx \mathbf{y}_{\ell, m}-\mathbf{f}\left(\overline{\mathbf{g}}_{\ell, m}\right)-\mathbf{F}_{\ell, m} \mathbf{G}_{\ell, m} \delta \mathbf{z} \\
& =\overline{\mathbf{e}}_{\ell, m}-\mathbf{H}_{\ell, m} \delta \mathbf{z}
\end{aligned}
$$

correct to first order, where

$$
\begin{aligned}
\overline{\mathbf{e}}_{\ell, m} & :=\mathbf{y}_{\ell, m}-\mathbf{f}\left(\overline{\mathbf{g}}_{\ell, m}\right), \\
\mathbf{H}_{\ell, m} & :=\mathbf{F}_{\ell, m} \mathbf{G}_{\ell, m}, \quad \mathbf{F}_{\ell, m}:=\left.\frac{\partial \mathbf{f}}{\partial \mathbf{g}}\right|_{\overline{\mathbf{g}}_{\ell, m}} .
\end{aligned}
$$

In the case that a loop closure is present in the pose chain between the initial landmark observation time, $t_{\ell}$, and the measurement time, $t_{m}$, the formulation changes slightly. Using the same perturbation scheme as in the derivation of (45), it is straightforward to show that

$$
\mathbf{G}_{\ell, m}:=\left[\left(\overline{\mathbf{T}}_{t_{m}, t_{\ell}} \overline{\mathbf{p}}_{\ell}\right) \odot\left(\int_{t_{2}}^{t_{m}} \overline{\mathcal{T}}_{t_{m}, \tau} \boldsymbol{\Psi}(\tau) d \tau+\int_{t_{\ell}}^{t_{1}} \overline{\mathcal{T}}_{t_{m}, \tau} \boldsymbol{\Psi}(\tau) d \tau\right) \quad\left(\overline{\mathbf{T}}_{t_{m}, t_{\ell}} \overline{\mathbf{p}}_{\ell}\right) \odot \overline{\mathcal{T}}_{t_{m}, t_{2}} \quad \overline{\mathbf{T}}_{t_{m}, t_{\ell}} \mathbf{D} \mathbf{P}_{\ell}\right]
$$

where the middle term relates to the loop-closure perturbation $\delta \boldsymbol{\xi}_{t_{2}, t_{1}}^{\mathrm{LC}}$, in $\delta \mathbf{z}=\left[\begin{array}{llll}\delta \mathbf{c}^{T} & \delta \boldsymbol{\xi}_{t_{2}, t_{1}}^{\mathrm{LC}} & \delta \boldsymbol{\zeta}^{T}\end{array}\right]^{T}$.

\subsubsection{Gauss-Newton Algorithm}

Setting $\frac{\partial^{T} J}{\partial \delta \mathbf{z}}=\mathbf{0}$, we find the optimal state update equation for a single iteration of Gauss-Newton:

$$
\left(\mathbf{A}_{s}+\mathbf{A}_{u}\right) \delta \mathbf{z}^{*}=\mathbf{b}_{s}+\mathbf{b}_{u} .
$$




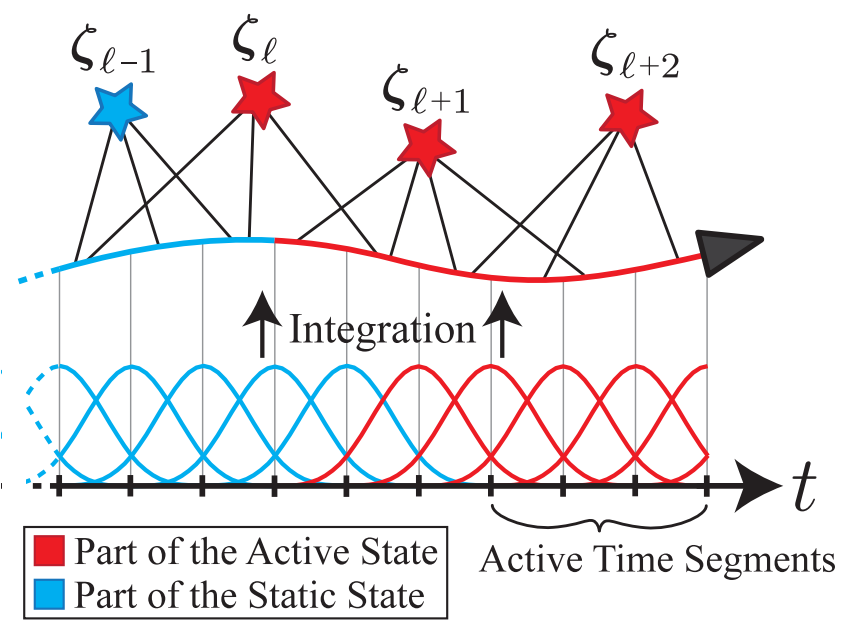

Figure 9: This figure depicts the use of active state parameters in a window-style filter. For illustration, uniformly weighted cubic B-spline basis functions, that have a local support of four, are used to portray the parametric velocity profile estimate. The integrated robot trajectory, shown above the velocity profile, is depicted to align on the time axis, with the landmarks observed at the connected timestamps. Determining the active state begins by defining the active time segments; in discrete terms, this is comparable to determining which poses to optimize in a window-style filter. Any basis function that influences the active time segments becomes part of the active state. A landmark position becomes part of the active state if it is observed at a time that the robot trajectory is affected by changes to the active basis function weights. Note that some of the static basis function weights are still required in order to calculate expected measurements for landmarks with observations from both affected and unaffected time intervals (e.g., $\boldsymbol{\zeta}_{\ell}$ ).

With respect to $J_{s}, \frac{\partial^{T} J_{s}}{\partial \delta \mathbf{z}}=\mathbf{A}_{s} \delta \mathbf{z}-\mathbf{b}_{s}$, where,

$$
\begin{aligned}
\mathbf{A}_{s} & :=\sum_{\ell, m} \mathbf{H}_{\ell, m}^{T} \mathbf{R}_{\ell, m}^{-1} \mathbf{H}_{\ell, m}, \\
\mathbf{b}_{s} & :=\sum_{\ell, m} \mathbf{H}_{\ell, m}^{T} \mathbf{R}_{\ell, m}^{-1} \overline{\mathbf{e}}_{\ell, m} .
\end{aligned}
$$

As in Furgale et al. (2012), we find that $\frac{\partial^{T} J_{u}}{\partial \delta \mathbf{z}}=\mathbf{A}_{u} \delta \mathbf{z}-\mathbf{b}_{u}$, where,

$$
\begin{aligned}
\mathbf{A}_{u} & :=\left[\begin{array}{cc}
\int_{0}^{T} \dot{\Psi}(\tau)^{T} \mathbf{Q}^{-1} \dot{\Psi}(\tau) d \tau & \mathbf{0} \\
\mathbf{0} & \mathbf{0}
\end{array}\right], \\
\mathbf{b}_{u} & :=-\mathbf{A}_{u} \overline{\mathbf{z}} .
\end{aligned}
$$

Note that the term $\mathbf{A}_{u}$ only needs to be calculated once, as it does not depend on the state parameters. Following the GaussNewton approach, the solution to $\delta \mathbf{z}^{*}$ is used to update the nominal solutions iteratively and to convergence. The pertinent state update equations are: $\overline{\mathbf{c}} \leftarrow \overline{\mathbf{c}}+\delta \mathbf{c}^{*}, \overline{\boldsymbol{\zeta}} \leftarrow \overline{\boldsymbol{\zeta}}+\delta \boldsymbol{\zeta}^{*}$, and in the case of a loop closure, $\overline{\mathbf{T}}_{t_{2}, t_{1}}^{\mathrm{LC}} \leftarrow \exp \left(\left(\delta \boldsymbol{\xi}_{t_{2}, t_{1}}^{L C}\right)^{\wedge}\right) \overline{\mathbf{T}}_{t_{2}, t_{1}}^{\mathrm{LC}}$.

\subsubsection{Implementation Efficiency}

There are three major computational costs associated with running this estimator. The first major cost is the numerical integrations of (11) and (46b). We note from the derivation of (32) that the integral term in $\mathbf{G}_{\ell, m}$ can be written as

$$
\int_{t_{\ell}}^{t_{m}} \overline{\mathcal{T}}_{t_{m}, \tau} \mathbf{\Psi}(\tau) d \tau=\operatorname{Ad}\left(\overline{\mathbf{T}}\left(t_{m}\right)\right)\left(\mathbf{I}\left(t_{m}\right)-\mathbf{I}\left(t_{\ell}\right)\right)
$$

where,

$$
\mathbf{I}(t):=\int_{0}^{t} \operatorname{Ad}(\overline{\mathbf{T}}(\tau))^{-1} \mathbf{\Psi}(\tau) d \tau
$$


Given that many of the required integral calculations will overlap, we aim to compute all the integral terms efficiently by conducting a single pass over the entire time period that we are currently optimizing. In the full batch optimization, the following equations are used iteratively for $t=[0, T]$,

$$
\begin{aligned}
\overline{\mathbf{T}}(t+\Delta t) & \leftarrow \exp \left((\Delta t \cdot \mathbf{\Psi}(t) \overline{\mathbf{c}})^{\wedge}\right) \overline{\mathbf{T}}(t), \\
\mathbf{I}(t+\Delta t) & \leftarrow \mathbf{I}(t)+\Delta t \cdot \operatorname{Ad}(\overline{\mathbf{T}}(t))^{-1} \mathbf{\Psi}(t),
\end{aligned}
$$

where $\overline{\mathbf{T}}(0)=\mathbf{1}$ and $\mathbf{I}(0)=\mathbf{0}$. The integration step, $\Delta t$, is chosen so that we have intermediate terms for $\overline{\mathbf{T}}$ and $\mathbf{I}$ at each measurement time, $t_{\ell, m}$. By storing the intermediate steps, we can compute $\overline{\mathbf{T}}_{t_{m}, t_{\ell}}$ and $\mathbf{G}_{\ell, m}$ for each measurement.

The second major cost in this estimator is building the matrix $\mathbf{A}_{s}$. This step can be exceptionally expensive depending on the choice of basis functions used to parameterize $\boldsymbol{\Psi}(t)$. Specifically, it is highly beneficial to use a basis function type that has local support. At any given time, we want only a subset of the basis functions, $\boldsymbol{\psi}_{b}(t)$, to be non-zero. Using local support introduces an exploitable sparsity to the matrix $\boldsymbol{\Psi}(t)$. As mentioned in Section 3.3.1, our implementation of the estimator uses cubic B-spline basis functions, because they have local support in addition to simple analytical integrals and derivatives.

The third cost is related to solving the linear system of equations in (52). To do this efficiently, we note that it can be written using the $2 \times 2$ block structure,

$$
\left[\begin{array}{ll}
\mathbf{A}_{11} & \mathbf{A}_{12} \\
\mathbf{A}_{12}^{T} & \mathbf{A}_{22}
\end{array}\right]\left[\begin{array}{l}
\delta \boldsymbol{\theta}^{*} \\
\delta \boldsymbol{\zeta}^{*}
\end{array}\right]=\left[\begin{array}{l}
\mathbf{b}_{1} \\
\mathbf{b}_{2}
\end{array}\right] .
$$

The relative formulation of the problem and addition of the motion model change the traditional block-diagonal sparsity pattern of $\mathbf{A}_{11}$. However, since $\mathbf{A}_{22}$ is still block-diagonal and is typically much larger than $\mathbf{A}_{11}$, we may apply the usual Schur complement in the typical bundle adjustment fashion with no additional cost:

$$
\left[\begin{array}{cc}
\mathbf{A}_{11}-\mathbf{A}_{12} \mathbf{A}_{22}^{-1} \mathbf{A}_{12}^{T} & \mathbf{0} \\
\mathbf{A}_{12}^{T} & \mathbf{A}_{22}
\end{array}\right]\left[\begin{array}{l}
\delta \boldsymbol{\theta}^{*} \\
\delta \boldsymbol{\zeta}^{*}
\end{array}\right]=\left[\begin{array}{c}
\mathbf{b}_{1}-\mathbf{A}_{12} \mathbf{A}_{22}^{-1} \mathbf{b}_{2} \\
\mathbf{b}_{2}
\end{array}\right] .
$$

Using the system above, it is simple to solve for $\delta \boldsymbol{\theta}^{*}$ while exploiting the block-diagonal sparity of $\mathbf{A}_{22}$ to find $\mathbf{A}_{22}^{-1}$ efficiently. Back-substituting the solution for $\delta \boldsymbol{\theta}^{*}$, we can then quickly calculate the landmark updates, $\delta \boldsymbol{\zeta}^{*}$.

\subsubsection{Window Filter}

In order to run the estimator online we optimize over a subset of the basis functions used in the time segments leading up to the most recent measurement. However, in order for this technique to achieve constant-time performance, the subset of basis functions must have temporally local effects on the robot trajectory, $\varpi(t)$. For this to be true, it is a requirement that the basis functions used in the estimator have local support.

Before setting up the local batch optimization problem, we begin by identifying the active state, illustrated in Figure 9. The active subset of basis functions and all landmarks measured during the affected time period are added to a list of active parameters that will be updated in the optimization. All measurements of these active landmark parameters are included when building the terms $\mathbf{A}_{s}$ and $\mathbf{b}_{s}$. This may require the full nominal solution, $\overline{\mathbf{c}}$, as some of the static basis function coefficients may be required to evaluate $\bar{\varpi}(t)$ near and outside the bounds of the active time period.

In the case of a loop closure, the active state selection changes slightly to include both the discrete loop-closure transformation, and temporal basis functions on both sides of the spatial link; the basis functions that are made active are the ones that overlap in time with any of the landmark observations that were matched across the loop closure. All measurements taken during the affected time periods are included in the optimization and landmarks matched across the loop closure are merged.

\subsubsection{Pose-Graph Relaxation}

Although the relative SLAM problem formulation argues that a global map is not necessary for many common robotic tasks, a global map may be desirable or required in some situations; for example, human inspection of a large-scale map, or humanin-the-loop decisions, such as macro-level path planning or goal setting.

In order to generate a globally consistent SLAM solution in a computationally tractable manner, a common practice is to implement pose-graph relaxation or pose-graph SLAM; the derivation of which is well known (Olson et al., 2006; Grisetti et al., 2007). The general concept of the optimization is to use the estimated discrete relative pose transforms as measurements in a new problem that aims to solve for the global poses. 
In order to relax the relative continuous-time trajectory into global coordinates, we first discretize our solution into a set of discrete relative transforms, $\mathbf{T}_{t_{d+1}, t_{d}}$, which occur between times $t_{d}$ and $t_{d+1}$, for $d=1 \ldots D$. Obtaining the relative transforms is straightforward using the integration scheme in (11),

$$
\mathbf{T}_{t_{d+1}, t_{d}}=\mathbf{T}\left(t_{d+1}\right) \mathbf{T}\left(t_{d}\right)^{-1}=\mathbf{1}+\int_{t_{d}}^{t_{d+1}} \varpi(\tau)^{\wedge} \mathbf{T}(\tau) d \tau
$$

However, obtaining the uncertainty of the relative pose estimates, $\boldsymbol{\Sigma}_{d+1, d}:=E\left[\delta \boldsymbol{\xi}_{t_{d+1}, t_{d}} \delta \boldsymbol{\xi}_{t_{d+1}, t_{d}}^{T}\right]$, involves some additional derivation. Note that the result from Barfoot and Furgale (2014) on compounding poses using fourth-order terms is not directly applicable to finding the uncertainty of the transform, $\mathbf{T}_{t_{d+1}, t_{d}}$, because $\delta \boldsymbol{\xi}\left(t_{d}\right)$ and $\delta \boldsymbol{\xi}\left(t_{d+1}\right)$ are correlated.

Using the relationship in (33) it is straightforward to see that

$$
\delta \boldsymbol{\xi}_{t_{d+1}, t_{d}} \approx\left(\overline{\mathcal{T}}\left(t_{d+1}\right) \int_{t_{d}}^{t_{d+1}} \overline{\mathcal{T}}(\tau)^{-1} \delta \varpi(\tau) d \tau\right)
$$

and therefore,

$$
\begin{aligned}
\boldsymbol{\Sigma}_{t_{d+1}, t_{d}} & =E\left[\delta \boldsymbol{\xi}_{t_{d+1}, t_{d}} \delta \boldsymbol{\xi}_{t_{d+1}, t_{d}}^{T}\right] \\
& \approx E\left[\left(\overline{\mathcal{T}}\left(t_{d+1}\right) \int_{t_{d}}^{t_{d+1}} \overline{\mathcal{T}}(\tau)^{-1} \delta \varpi(\tau) d \tau\right)\left(\overline{\mathcal{T}}\left(t_{d+1}\right) \int_{t_{d}}^{t_{d+1}} \overline{\mathcal{T}}\left(\tau^{\prime}\right)^{-1} \delta \varpi\left(\tau^{\prime}\right) d \tau^{\prime}\right)^{T}\right] \\
& =\overline{\mathcal{T}}\left(t_{d+1}\right) \int_{t_{d}}^{t_{d+1}} \int_{t_{d}}^{t_{d+1}} \overline{\mathcal{T}}(\tau)^{-1} E\left[\delta \varpi(\tau) \delta \varpi\left(\tau^{\prime}\right)^{T}\right] \overline{\mathcal{T}}\left(\tau^{\prime}\right)^{-T} d \tau^{\prime} d \tau \overline{\mathcal{T}}\left(t_{d+1}\right)^{T} .
\end{aligned}
$$

In practice, because we have parameterized the solution using basis functions, $\delta \varpi(t)=\mathbf{\Psi}(t) \delta \mathbf{c}$, it is convenient to compute the covariance as follows,

$$
\boldsymbol{\Sigma}_{t_{d+1}, t_{d}} \approx\left(\overline{\mathcal{T}}\left(t_{d+1}\right) \int_{t_{d}}^{t_{d+1}} \overline{\mathcal{T}}(\tau)^{-1} \mathbf{\Psi}(\tau) d \tau\right) E\left[\delta \mathbf{c} \delta \mathbf{c}^{T}\right]\left(\overline{\mathcal{T}}\left(t_{d+1}\right) \int_{t_{d}}^{t_{d+1}} \overline{\mathcal{T}}\left(\tau^{\prime}\right)^{-1} \mathbf{\Psi}\left(\tau^{\prime}\right) d \tau^{\prime}\right)^{T}
$$

where $E\left[\delta \mathbf{c} \delta \mathbf{c}^{T}\right]$ can be evaluated using part of the solution from our Gauss-Newton optimization problem in (59), after convergence. In an open-loop optimization, we know

$$
\operatorname{cov}\left(\delta \boldsymbol{\theta}^{*}, \delta \boldsymbol{\theta}^{*}\right)=E\left[\delta \mathbf{c}^{*} \delta \mathbf{c}^{* T}\right]=\left(\mathbf{A}_{11}-\mathbf{A}_{12} \mathbf{A}_{22}^{-1} \mathbf{A}_{12}^{T}\right)^{-1},
$$

and in a closed-loop optimization,

$$
\operatorname{cov}\left(\delta \boldsymbol{\theta}^{*}, \delta \boldsymbol{\theta}^{*}\right)=\left[\begin{array}{cc}
E\left[\delta \mathbf{c}^{*} \delta \mathbf{c}^{* T}\right] & E\left[\delta \mathbf{c}^{*} \delta \boldsymbol{\xi}_{t_{2}, t_{1}}^{L C}{ }^{* T}\right] \\
E\left[\delta \boldsymbol{\xi}_{t_{2}, t_{1}}^{L C}{ }^{*} \delta \mathbf{c}^{* T}\right] & E\left[\delta \boldsymbol{\xi}_{t_{2}, t_{1}}^{L C}{ }^{*} \delta \boldsymbol{\xi}_{t_{2}, t_{1}}^{L C}\right.
\end{array}\right]=\left(\mathbf{A}_{11}-\mathbf{A}_{12} \mathbf{A}_{22}^{-1} \mathbf{A}_{12}^{T}\right)^{-1},
$$

where $E\left[\delta \boldsymbol{\xi}_{t_{2}, t_{1}}^{L C} \delta \boldsymbol{\xi}_{t_{2}, t_{1}}^{L C}{ }^{* T}\right]$ is the uncertainty associated with the discrete loop closure, $\mathbf{T}_{t_{2}, t_{1}}^{L C}$.

\section{Outlier Rejection for Motion-Distorted 3D Visual Sensors}

In this section, we derive the motion-compensated RANSAC algorithm using a constant-velocity model. We show how our algorithm differs from the traditional rigid one and how using a velocity model impacts the algorithm. Furthermore, we propose two approximations to increase the computational efficiency of using a constant-velocity model.

\subsection{Problem Formulation}

For the feature correspondence problem, we define two sets of corresponding measurements, $\mathbf{y}_{m, 1}$ and $\mathbf{y}_{m, 2}$, where $m=$ $1 \ldots M$. Each measurement pair, $m$, is extracted from sequential images 1 and 2 at times, $t_{m, 1}$ and $t_{m, 2}$, with a temporal difference of $\Delta t_{m}:=t_{m, 2}-t_{m, 1}$. The sensor models for these measurements are

$$
\begin{aligned}
& \mathbf{y}_{m, 1}:=\mathbf{f}\left(\mathbf{T}\left(t_{m, 1}\right) \mathbf{p}_{m}\right)+\mathbf{n}_{m, 1}, \\
& \mathbf{y}_{m, 2}:=\mathbf{f}\left(\mathbf{T}\left(t_{m, 2}\right) \mathbf{p}_{m}\right)+\mathbf{n}_{m, 2},
\end{aligned}
$$




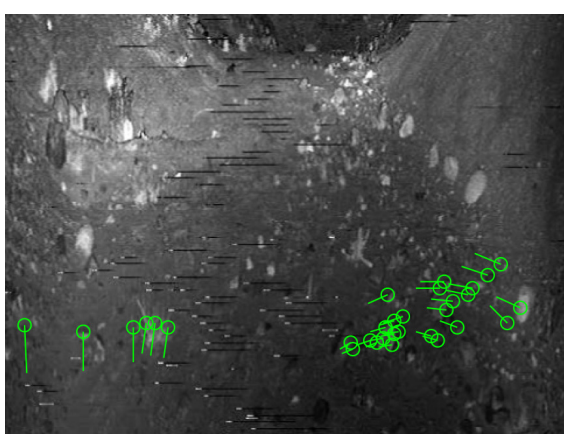

Figure 10: This figure shows the inlying feature tracks after applying the rigid RANSAC algorithm to our lidar intensity/range data. Due to fast motion and a slow vertical scan, only a small temporal band of the features are matched.

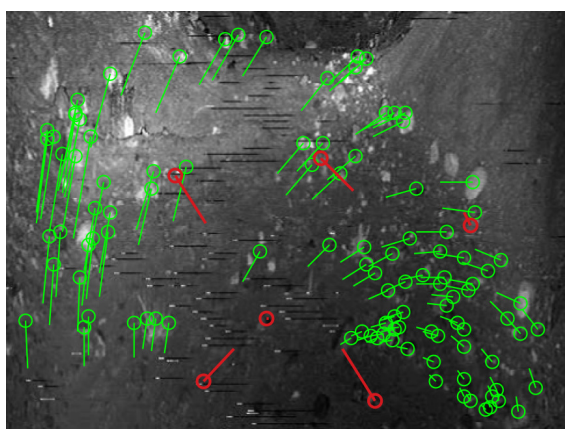

Figure 11: This figure shows that relaxing the measurement error threshold for the rigid RANSAC algorithm allows for a larger number of inlying matches (green), but also introduces the possibility of outliers (red).

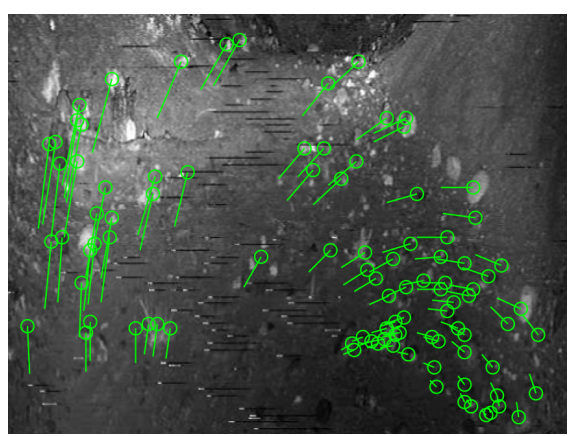

Figure 12: This figure shows the inlying matches after applying the MCRANSAC algorithm; by using a constant velocity to model the motion of the sensor, the filter is able to better account for the distortion of the image.

where $\mathbf{f}(\cdot)$ is the nonlinear camera model, $\mathbf{T}(t)$ is the $4 \times 4$ homogeneous transform matrix that specifies the pose of the sensor frame, $\underline{\mathcal{F}}_{s}(t)$, with respect to the inertial frame, $\underline{\mathcal{F}}_{i}$, at time $t$, and the measurement noises, $\mathbf{n}_{m, 1} \sim \mathcal{N}\left(\mathbf{0}, \mathbf{R}_{m, 1}\right)$ and $\mathbf{n}_{m, 2} \sim \mathcal{N}\left(\mathbf{0}, \overrightarrow{\mathbf{R}}_{m, 2}\right)$, are assumed to be normally distributed with covariances $\mathbf{R}_{m, 1}$ and $\mathbf{R}_{m, 2}$. Note that each measurement pairing, $m$, is simply the hypothesis of a common landmark, typically based on the similarity of appearance-based feature descriptors, and may not actually be projected from the same 3D location, $\mathbf{p}_{m}:=\left[\begin{array}{ll}\boldsymbol{\zeta}_{m}^{T} & 1\end{array}\right]^{T}$. The goal is to determine the subset of all $M$ measurement pairs, $\mathbf{y}_{m, 1}$ and $\mathbf{y}_{m, 2}$, that make up the most likely set of inliers.

The structure of the RANSAC algorithm consists of only a few main steps. It begins by selecting $N$ random subsets of $S$ measurement pairs, where $S$ is the minimum number of pairs needed to solve the mathematical model. When using 3D point correspondences, such as in stereo camera or lidar data, $S$ is typically 3 . The number of required iterations, $N$, can be chosen using the equation

$$
N=\frac{\ln \left(1-p_{\text {succ }}\right)}{\ln \left(1-p_{\text {in }}^{S}\right)}
$$

where $p_{\text {succ }}$ is the probability of choosing $S$ measurement pairs that are all inliers and $p_{\text {in }}$ is the probability of a single pair, $m$, being an inlier. The first step, for each of the $N$ subsets, is to solve the mathematical model that can be used to find the unknown pose change of the sensor frame, $\mathbf{T}_{m}$, between times $t_{m, 1}$ and $t_{m, 2}$,

$$
\mathbf{T}_{m}:=\mathbf{T}\left(t_{m, 2}\right) \mathbf{T}\left(t_{m, 1}\right)^{-1} .
$$

The second step is to then apply the calculated transform, $\mathbf{T}_{m}$, to the measurements $\mathbf{y}_{m, 1}$, using

$$
\hat{\mathbf{p}}_{m, 2}:=\mathbf{T}_{m} \mathbf{p}_{m, 1}, \quad \mathbf{p}_{m, 1}:=\mathbf{f}^{-1}\left(\mathbf{y}_{m, 1}\right),
$$

where the calculation of $\mathbf{p}_{m, 1}$ is independent of the mathematical model and is performed only once for all $M$ pairs. Note we take advantage of the invertible camera model available to 3D sensors. This assumption prevents us from directly applying our method to a monocular rolling-shutter camera; however, with some special care we believe it is possible to extend our technique for the monocular case. The transformed measurements, $\hat{\mathbf{p}}_{m, 2}$, are then reprojected back into measurement space:

$$
\hat{\mathbf{y}}_{m, 2}:=\mathbf{f}\left(\hat{\mathbf{p}}_{m, 2}\right) .
$$

Finally, each model is evaluated by finding the number of measurements pairs, $\mathbf{y}_{m, 1}$ and $\mathbf{y}_{m, 2}$, that satisfy

$$
\left(\mathbf{y}_{m, 2}-\hat{\mathbf{y}}_{m, 2}\right)^{T} \mathbf{R}_{m, 2}^{-1}\left(\mathbf{y}_{m, 2}-\hat{\mathbf{y}}_{m, 2}\right)<\alpha,
$$

where $\alpha$ is a threshold on normalized reprojection error. For simplicity, the model with the highest number of inliers is chosen. 


\subsection{Classic Rigid RANSAC}

The mathematical model used in the classic RANSAC algorithm is a single rigid pose change between two static frames. It does not take into consideration the possibility of individual measurement times, and in order to use it, we must approximate our motion-distorted measurements as occurring at nominal image times, $\bar{t}_{1}$ and $\bar{t}_{2}$. The approximation being made is that

$$
\Delta t=\bar{t}_{2}-\bar{t}_{1} .
$$

The transform, $\mathbf{T}_{m}$, is therefore the same for all pairs, $m$, and can be solved with only three non-collinear point pairs, in closed form, using the algorithms presented in Horn (1987), Arun et al. (1987), or Umeyama (1991). This assumption is reasonable for rolling-shutter-type images that have little to no distortion. However, under significant motion it quickly becomes clear that the rigid algorithm is unfit for identifying a good set of inliers.

Setting the threshold on reprojection error, $\alpha$, appropriately, the rigid RANSAC algorithm is limited to finding inliers that have a similar temporal difference, $\Delta t$. In practice, we tuned $\alpha$ to find as many inliers as possible (over the whole trajectory), without including outliers at standstill. Due to the slow vertical scan of our sensor, it is only possible to match a temporal band of features at high vehicle speeds, as seen Figure 10. Loosening $\alpha$ to allow for more feature matches at high vehicle speeds also allows for the inclusion of outliers, as seen in Figure 11.

\subsection{Motion-Compensated RANSAC}

To compensate for motion during image capture, it is proposed that we can approximate the motion of the sensor as a constant velocity, $\varpi:=\left[\begin{array}{ll}\boldsymbol{\nu}^{T} & \boldsymbol{\omega}^{T}\end{array}\right]^{T}$, where $\boldsymbol{\nu}$ and $\boldsymbol{\omega}$ are the linear and angular components, expressed in the sensor frame (Anderson and Barfoot, 2013a). In order to calculate $\hat{\mathbf{y}}_{m, 2}$, we derive the relationship between the sensor velocity, $\varpi$, and the transform $\mathbf{T}_{m}$. Assuming constant-velocity, the desired transform is simply

$$
\mathbf{T}_{m}=\exp \left(\Delta t_{m} \varpi^{\wedge}\right) .
$$

The major difference in moving from a rigid model to a constant-velocity model is that there now exists a different pose change for each measurement pair, based on their temporal difference, $\Delta t_{m}$.

\subsubsection{Nonlinear Least-Squares Estimator}

For each of the $N$ subsets, the first major RANSAC step is to solve for the optimal constant velocity, $\varpi$, that minimizes the $S$ paired measurements' reprojection error. To do this, we setup a typical nonlinear least-squares estimation scheme, similar to that of a bundle adjustment problem. The objective function that we wish to minimize is simply

$$
J(\varpi):=\frac{1}{2} \sum_{m=1}^{M} \mathbf{e}_{m}^{T} \mathbf{R}_{m, 2}^{-1} \mathbf{e}_{m},
$$

where the error term is defined as

$$
\mathbf{e}_{m}:=\mathbf{y}_{m, 2}-\hat{\mathbf{y}}_{m, 2}=\mathbf{y}_{m, 2}-\mathbf{f}\left(\mathbf{T}_{m} \mathbf{p}_{m, 1}\right) .
$$

Note that we avoid estimating the landmark positions by assuming perfect knowledge of $\mathbf{p}_{m, 1}$ (i.e., no noise on the measurement $\mathbf{y}_{m, 1}$ ). In order to linearize the measurement error, $\mathbf{e}_{m}$, we begin by noting the composition of two nonlinearities; the nonlinear camera model, and the transform $\mathbf{T}_{m}$. Starting with the transformation nonlinearity, we define

$$
\mathbf{g}_{m}(\varpi):=\mathbf{T}_{m} \mathbf{p}_{m, 1} .
$$

Consider the perturbation to the velocity,

$$
\mathbf{T}_{m}=\exp \left(\Delta t_{m} \varpi^{\wedge}\right)=\exp \left(\Delta t_{m}(\bar{\varpi}+\delta \varpi)^{\wedge}\right),
$$

where $\bar{\varpi}$ is the nominal solution and $\delta \varpi$ is the perturbation. Using the result from Barfoot and Furgale (2014, Appendix A),

$$
\exp \left((\boldsymbol{\xi}+\boldsymbol{\pi})^{\wedge}\right) \approx \exp \left((\mathcal{J}(\boldsymbol{\xi}) \boldsymbol{\pi})^{\wedge}\right) \exp \left(\boldsymbol{\xi}^{\wedge}\right),
$$


which is correct to first order in $\pi$, where

$$
\begin{aligned}
\mathcal{J}(\boldsymbol{\xi}):= & {\left[\begin{array}{cc}
\mathbf{J}(\boldsymbol{\phi}) & \mathbf{Q}(\boldsymbol{\xi}) \\
\mathbf{0} & \mathbf{J}(\boldsymbol{\phi})
\end{array}\right], \quad \boldsymbol{\xi}:=\left[\begin{array}{l}
\boldsymbol{\rho} \\
\boldsymbol{\phi}
\end{array}\right], } \\
\mathbf{Q}(\boldsymbol{\xi}):= & \frac{1}{2} \boldsymbol{\rho}^{\wedge}+\frac{\phi-\sin \phi}{\phi^{3}}\left(\boldsymbol{\phi}^{\wedge} \boldsymbol{\rho}^{\wedge}+\boldsymbol{\rho}^{\wedge} \boldsymbol{\phi}^{\wedge}+\boldsymbol{\phi}^{\wedge} \boldsymbol{\rho}^{\wedge} \boldsymbol{\phi}^{\wedge}\right) \\
& -\frac{1-\frac{\phi^{2}}{2}-\cos \phi}{\phi^{4}}\left(\boldsymbol{\phi}^{\wedge} \boldsymbol{\phi}^{\wedge} \boldsymbol{\rho}^{\wedge}+\boldsymbol{\rho}^{\wedge} \boldsymbol{\phi}^{\wedge} \boldsymbol{\phi}^{\wedge}-3 \boldsymbol{\phi}^{\wedge} \boldsymbol{\rho}^{\wedge} \boldsymbol{\phi}^{\wedge}\right) \\
& -\frac{1}{2}\left(\frac{1-\frac{\phi^{2}}{2}-\cos \phi}{\phi^{4}}-3 \frac{\phi-\sin \phi-\frac{\phi^{3}}{6}}{\phi^{5}}\right)\left(\phi^{\wedge} \boldsymbol{\rho}^{\wedge} \boldsymbol{\phi}^{\wedge} \boldsymbol{\phi}^{\wedge}+\boldsymbol{\phi}^{\wedge} \boldsymbol{\phi}^{\wedge} \boldsymbol{\rho}^{\wedge} \boldsymbol{\phi}^{\wedge}\right),
\end{aligned}
$$

we find that,

$$
\begin{aligned}
\mathbf{T}_{m} & \approx \exp \left(\Delta t_{m} \cdot\left(\overline{\mathcal{J}}_{m} \delta \varpi\right)^{\wedge}\right) \exp \left(\Delta t_{m} \cdot \bar{\varpi}^{\wedge}\right) \\
& =\exp \left(\Delta t_{m} \cdot\left(\overline{\mathcal{J}}_{m} \delta \varpi\right)^{\wedge}\right) \overline{\mathbf{T}}_{m}
\end{aligned}
$$

where $\overline{\mathcal{J}}_{m}:=\mathcal{J}\left(\Delta t_{m} \bar{\varpi}\right)$. This can be simplified again using the small-pose approximation found in (14),

$$
\mathbf{T}_{m} \approx\left(\mathbf{1}+\Delta t_{m} \cdot\left(\overline{\mathcal{J}}_{m} \delta \varpi\right)^{\wedge}\right) \overline{\mathbf{T}}_{m}
$$

Applying this perturbation scheme to (74),

$$
\begin{aligned}
\mathbf{g}_{m}(\overline{\boldsymbol{\varpi}}+\delta \varpi) & \approx\left(\mathbf{1}+\Delta t_{m}\left(\overline{\mathcal{J}}_{m} \delta \varpi\right)^{\wedge}\right) \overline{\mathbf{T}}_{m} \mathbf{p}_{m, 1} \\
& =\overline{\mathbf{T}}_{m} \mathbf{p}_{m, 1}+\Delta t_{m}\left(\overline{\mathcal{J}}_{m} \delta \varpi\right)^{\wedge} \overline{\mathbf{T}}_{m} \mathbf{p}_{m, 1}
\end{aligned}
$$

Using the homogeneous-coordinate identity in (48a), it follows that,

$$
\mathbf{g}_{m}(\bar{\varpi}+\delta \varpi)=\overline{\mathbf{g}}_{m}+\mathbf{G}_{m} \delta \varpi
$$

correct to first order, where,

$$
\begin{aligned}
\overline{\mathbf{g}}_{m} & :=\overline{\mathbf{T}}_{m} \mathbf{p}_{m, 1}, \\
\mathbf{G}_{m} & :=\Delta t_{m}\left(\overline{\mathbf{T}}_{m} \mathbf{p}_{m, 1}\right)^{\odot} \overline{\mathcal{J}}_{m} .
\end{aligned}
$$

Returning to our measurement error term,

$$
\begin{aligned}
\mathbf{e}_{m} & \approx \mathbf{y}_{m, 2}-\mathbf{f}\left(\overline{\mathbf{g}}_{m}+\mathbf{G}_{m} \delta \varpi\right) \\
& \approx \overline{\mathbf{e}}_{m}-\mathbf{H}_{m} \delta \varpi
\end{aligned}
$$

correct to first order, where

$$
\begin{aligned}
\overline{\mathbf{e}}_{m} & :=\mathbf{y}_{m, 2}-\mathbf{f}\left(\overline{\mathbf{g}}_{m}\right), \\
\mathbf{H}_{m} & :=\mathbf{F}_{m} \mathbf{G}_{m}, \quad \mathbf{F}_{m}:=\left.\frac{\partial \mathbf{f}}{\partial \mathbf{g}}\right|_{\overline{\mathbf{g}}_{m}} .
\end{aligned}
$$

Setting $\frac{\partial J}{\partial \delta \varpi^{T}}=\mathbf{0}$, we find the optimal state update equation for a single iteration of Gauss-Newton:

$$
\left(\sum_{m} \mathbf{H}_{m}^{T} \mathbf{R}_{m, 2}^{-1} \mathbf{H}_{m}\right) \delta \varpi^{*}=\sum_{m} \mathbf{H}_{m}^{T} \mathbf{R}_{m, 2}^{-1} \overline{\mathbf{e}}_{m}
$$

Using the normal iterative Gauss-Newton approach, we solve the system of equations for $\delta \varpi^{*}$, and update the nominal solution, $\bar{\varpi} \leftarrow+\delta \varpi^{*}$, until convergence. The estimator requires a minimum of three well-spaced point correspondences (see Appendix A). Note that three is only the minimum number of correspondences required, the estimator can also be used to improve the $\varpi$ estimate after finding a larger set of likely inliers. 


\subsubsection{Point Transformation}

In order to compare each of the $N$ constant-velocity models, we must evaluate the number of inliers. This is done by transforming each of the measurements, $\mathbf{y}_{m, 1}$, into the corresponding frame, $\underline{\mathcal{F}}_{s}\left(t_{m, 2}\right)$. The required transform, $\mathbf{T}_{m}$, can be calculated using (71). In contrast to the rigid RANSAC algorithm, which calculates only one transform per model, our motion-compensated RANSAC algorithm requires that $\mathbf{T}_{m}$ be evaluated for each pair, $m$, using each of the $N$ constantvelocity models.

\subsection{Fast Motion-Compensated RANSAC}

The computation of a transform, $\mathbf{T}_{m}$, for each measurement pair, $m$, using each of the $N$ constant-velocity models, adds a significant amount of overhead to the computational cost. Furthermore, the iterative estimator has to run for each of the $N$ randomly seeded measurement subsets. In order to improve the performance of the algorithm, we propose a Euclidean least-squares estimator and a heuristic for the point-transformation step.

\subsubsection{Euclidean Least-Squares Estimator}

We begin by reformulating the error in Euclidean space:

$$
\mathbf{e}_{m}:=\mathbf{p}_{m, 2}-\hat{\mathbf{p}}_{m, 2}, \quad \hat{\mathbf{p}}_{m, 2}=\mathbf{T}_{m} \mathbf{p}_{m, 1} .
$$

The advantage of this is that it eliminates the need to linearize the camera model. The disadvantage is that the algorithm is no longer optimizing with respect to the error that is evaluated in the inlier criterion (69).

Given that the two images are sequential, and relatively close in time, we propose the assumption that $\mathbf{T}_{m}$ is 'small', and therefore can be approximated using (14):

$$
\begin{aligned}
\mathbf{e}_{m} & \approx \mathbf{p}_{m, 2}-\left(\mathbf{1}+\Delta t_{m} \varpi^{\wedge}\right) \mathbf{p}_{m, 1} \\
& =\mathbf{p}_{m, 2}-\mathbf{p}_{m, 1}-\Delta t_{m} \mathbf{p}_{m, 1}^{\odot} \varpi \\
& =\mathbf{q}_{m}-\mathbf{Q}_{m} \varpi,
\end{aligned}
$$

where

$$
\begin{aligned}
\mathbf{q}_{m} & :=\mathbf{p}_{m, 2}-\mathbf{p}_{m, 1}, \\
\mathbf{Q}_{m} & :=\Delta t_{m} \mathbf{p}_{m, 1}^{\odot} .
\end{aligned}
$$

The simplified objective function that we minimize is

$$
J(\varpi):=\frac{1}{2} \sum_{m} \mathbf{e}_{m}^{T} \mathbf{e}_{m} .
$$

Inserting $\mathbf{e}_{m}$ into $J$ and setting $\frac{\partial J}{\varpi^{T}}=\mathbf{0}$,

$$
\left(\sum_{m} \mathbf{Q}_{m}^{T} \mathbf{Q}_{m}\right) \varpi=\sum_{m} \mathbf{Q}_{m}^{T} \mathbf{q}_{m},
$$

which can be solved for $\varpi$ in one step.

\subsubsection{Discretization of Required Transforms}

The motion-compensated problem formulation requires the calculation of the transform, $\mathbf{T}_{m}$, for each measurement pair, $m$, using each of the $N$ proposed constant-velocity models. In order to improve computational performance, it is proposed that for each of the $N$ models, finding only a discretized subset of the transforms is admissible. Based on the time differences, $\Delta t_{m}$, the measurement pairs, $m$, are sorted into a set of discrete bins, uniformly spaced between the minimum and maximum values of $\Delta t_{m}$; a transform is then calculated for each bin, based on its centre time. During the evaluation of each measurement pair, $m$, the transform $\mathbf{T}_{m}$ is simply approximated by the appropriate bin transform. 


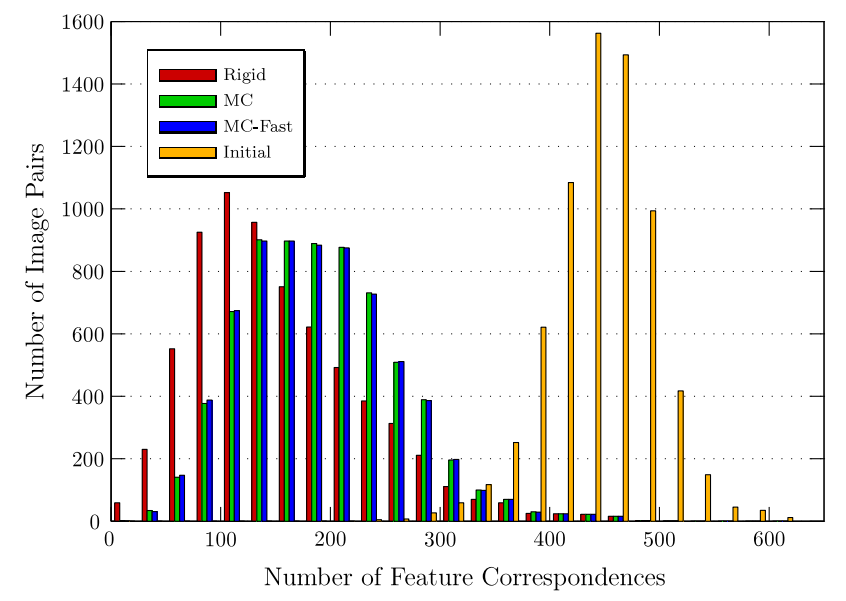

Figure 13: This figure shows the distribution of sequential image pairs over the number of successfully matched feature measurements. The distribution of 'initial' matches indicates the total number of hypothesized matches before filtering (including outliers). The rigid, motion-compensated, and fast motion-compensated filters are then applied to generate the plotted distribution of qualified inliers.

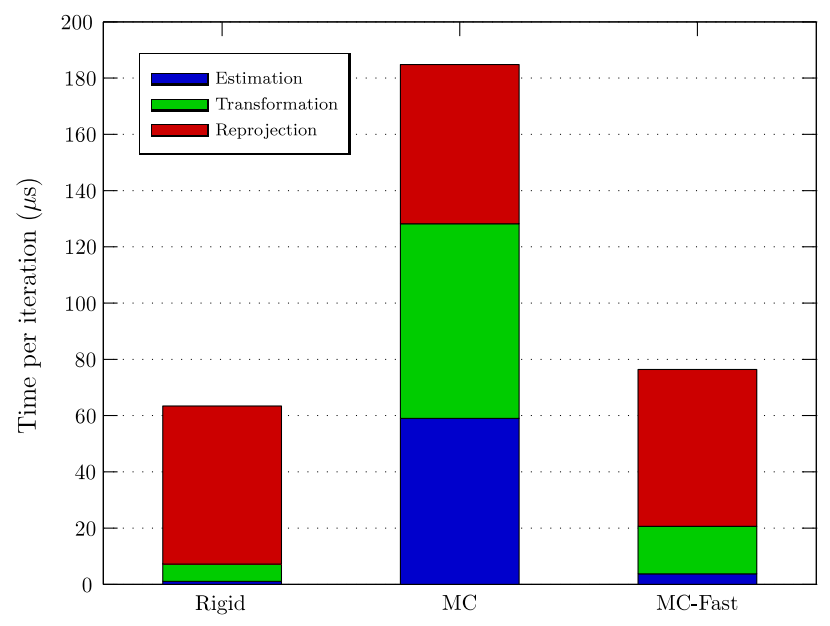

Figure 14: This figure shows the required computational effort of each RANSAC iteration. The iteration time is broken down into the three most demanding parts of the algorithm: the time taken to estimate the mathematical model (given 3 points), the time it takes to generate and apply the transform provided by the model, and the time it takes to reproject the Euclidean points through the spherical camera model. All RANSAC computations were performed in $\mathrm{C}++$ and timed with a $2.4 \mathrm{GHz}$ processor.

\section{Experimental Results}

In this section, we test and report results for both our relative continuous-time SLAM technique and motion-compensated RANSAC algorithm. The estimator and outlier rejection are tested using a set of real data that highlight our algorithm's ability to handle high-rate asynchronous measurements, while the robot undergoes motion in unstructured terrain.

\subsection{Dataset}

For the results presented in the paper, we make use of a $1.1 \mathrm{~km}$ and 6880 frame traversal from The Gravel Pit Lidar-Intensity Imagery Dataset (Anderson et al., 2012), which was acquired during a visual-teach-and-repeat experiment in Sudbury, Ontario, Canada, and used the ROC6 field robot, as seen in Figure 2. This experiment used an Autonosys LVC0702 lidar to capture azimuth, elevation, range, time, and intensity images, with a resolution of $480 \times 360$, at $2 \mathrm{~Hz}$, in unstructured, natural terrain. During the traversal, the robot travelled between 0 and $0.5 \mathrm{~m} / \mathrm{s}$. A GPU-accelerated SURF implementation was used to extract features from the 8-bit intensity imagery; azimuth, elevation, range, and time measurements are then interpolated for each feature and initial matches are determined using SURF feature descriptor similarity.

\subsection{Outlier Rejection}

\subsubsection{Quality}

The typical output of our motion-compensated RANSAC algorithm can be seen in Figure 12. In this section, we take two approaches to measuring the quality of the outlier-rejection schemes. First, we wish to evaluate the quantity of inliers that the algorithm is able to identify. Although we cannot know the true number of inliers between each pair of images, we can contrast algorithms by comparing the distribution of filtered feature correspondences against the initial distribution of possible feature matches. Assuming a relatively constant ratio between inliers and outliers across images, it is expected that the distributions should have a similar shape to the initial set, but a lower mean number of feature correspondences. The results of this distribution comparison can be seen in Figure 13. Note that both the motion-compensated and fast motion-compensated algorithms produce a near-identical result, which, as expected, is distinctly better than the rigid RANSAC algorithm's output. 

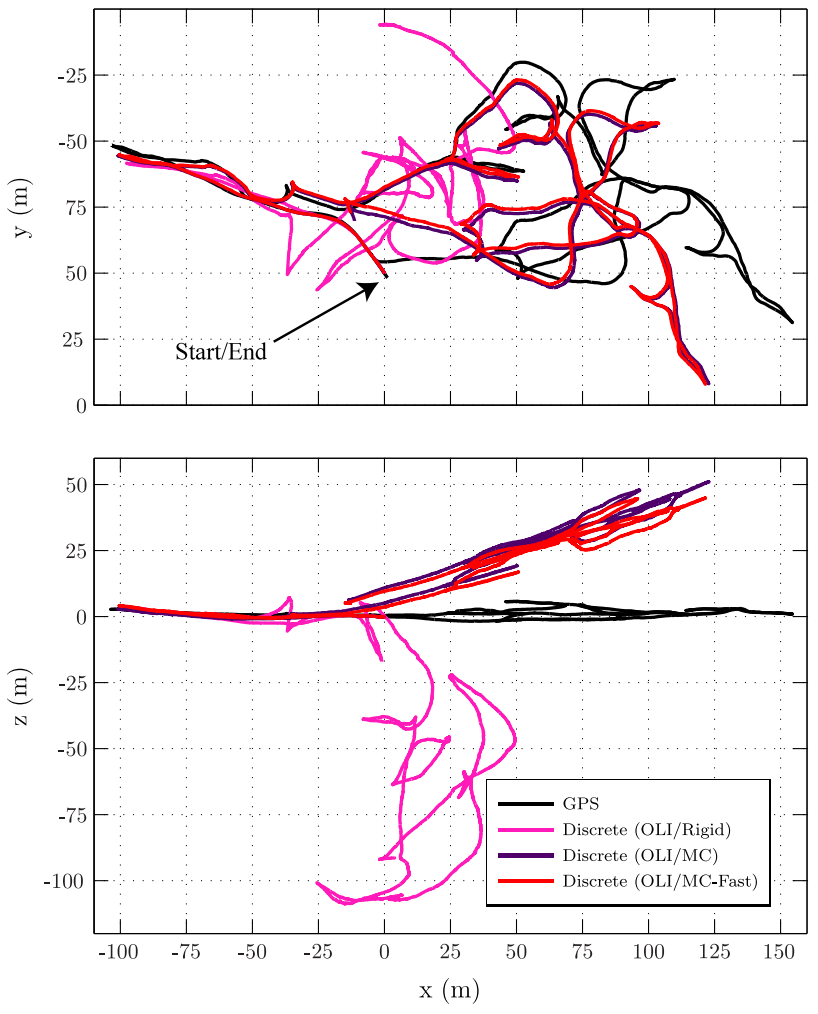

Figure 15: Top and side views of the robot trajectory over the whole $1.1 \mathrm{~km}$ dataset. The relative discrete-time bundle adjustment estimator used an active window size of 6 image stacks. DGPS tracks are in black, and the open-loop integration (OLI) of the discrete relative bundle adjustment solution is shown in different colours for various RANSAC outlier rejection schemes. Note that in order to make the discrete bundle adjustment state tractable and solvable, we assume no motion distortion in the images.
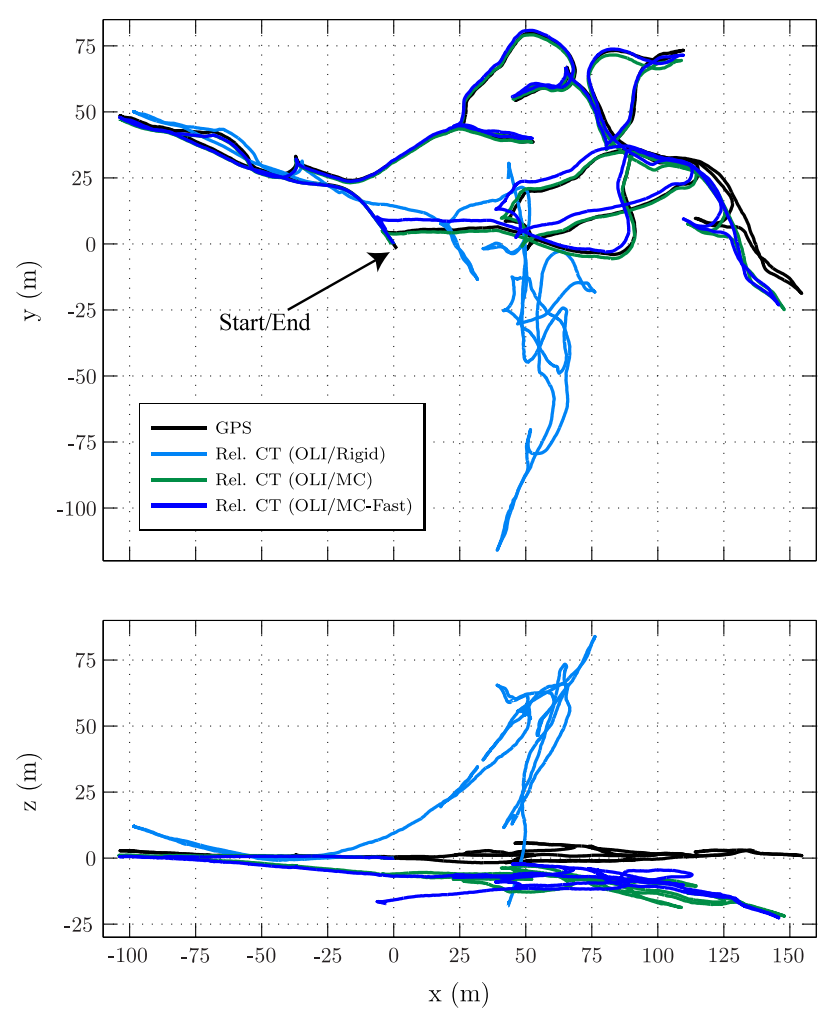

Figure 16: Top and side views of the robot trajectory for the relative continuous-time estimator over the whole $1.1 \mathrm{~km}$ dataset. The estimator used a knot spacing of 0.65 seconds, and an active window size of 5 time segments. Again, DGPS tracks are in black, and the open-loop integration (OLI) of the estimated velocity profile is shown in different colours for various RANSAC outlier rejection schemes. Note the significant improvement in contrast to the discrete solutions seen in Figure 15.

The second method used to evaluate the quality of outlier rejection is the output of the discrete and continuous-time bundleadjustment-style SLAM algorithms. The output of each RANSAC algorithm was used to initialize a set of open-loop feature correspondences; the window-style batch estimator was then run over each set of matches to produce an odometry estimate that can be compared to GPS ground truth. The output of the SLAM algorithms can be seen in Figures 15 and 16; further analysis of the relative continuous-time SLAM algorithm will be conducted in later sections, the important result to see for outlier rejection is the dramatic performance improvement between solutions using rigid and motion-compensated outlier rejection schemes.

Due to the 'slow' vertical scan of the lidar, the rigid RANSAC algorithm tends to find horizontal bands of temporally similar features; without a strong distribution of features in the vertical direction, it is expected that the pitch parameter of the estimation will suffer.

\subsubsection{Computational Efficiency}

In order to characterize the computational efficiency of each of these algorithms, the time spent completing each major RANSAC step was recorded over 1500 iterations, for each of the 6880 frames and averaged, as seen in Figure 14. The iteration time is broken down into three major sections: the time taken to estimate the mathematical model, the time it takes to generate and apply the transform, $\mathbf{T}_{m}$, to each measurement, and the time it takes to reproject the Euclidean points into 


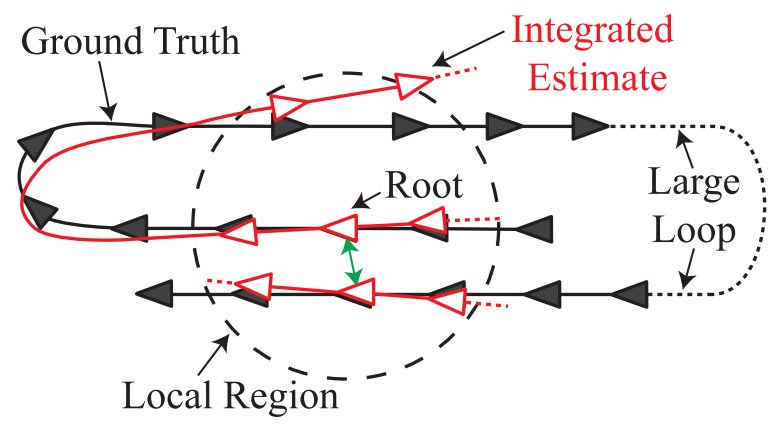

Figure 17: Illustration of the local RMS error calculation for a single root node. Note that open-loop integration (OLI) outside of the local region is needed to find the pose of the top two nodes, while closed-loop integration (CLI) can be used to quickly find the poses of the lower nodes.

spherical coordinates.

The classic rigid RANSAC algorithm is the fastest, as expected; however, quantitative analysis suggests that the rigid algorithm is completely unfit for use with this sensor. Moving to the motion-compensated RANSAC algorithm, the large increase in estimation time is due to the addition of the iterative Gauss-Newton process. The increase in transformation time is due to the cost of calculating a transformation matrix for each measurement pair. The fast motion-compensated RANSAC algorithm shows a drastic reduction in estimation time, although still not as fast as the rigid algorithm. For the transformation step, eight discrete transformations were used to approximate the constant-velocity model, which provided a significant speed up, with minimal cost to quality.

Note that the reprojection time is constant between all algorithms and is included purely to provide context to the total iteration time. Furthermore, note that the reprojection process can be ignored if the measurements are compared in Euclidean space. However, setting the threshold value appropriately is not as straightforward.

\subsection{Continuous-Time SLAM}

\subsubsection{Local Accuracy Metric}

Before discussing the results of the estimator, we begin by introducing the average local-area root-mean-square (ALARMS) error, a new metric similar to the one introduced by Sibley et al. (2010), which we use to evaluate local metric accuracy. The ALARMS error calculation is illustrated in Figure 17, and proceeds as follows: for each ground-truth node,

1. Search the ground truth (GPS) for all other nodes within some specified distance of the current (root) node

2. Perform a breadth first search (BFS) using the available topological information, starting with the root node, to determine the shortest paths to all other local nodes

3. Integrate along the found paths to obtain the pose of local nodes in the root node's coordinate frame

4. Fix the root node's pose to ground truth; in the absence of global orientation information, align using all the integrated local node positions and ground truth counterparts

5. Compute RMS Euclidean error for the local region subgraph

The ALARMS error is then the average of all the calculated RMS errors terms, where each error term is generated by using one of the ground-truth nodes to form a local region. In the absence of loop closures, only temporal links contribute to the topological connectivity of the graph.

\subsubsection{Open-Loop Solution}

From the 6880 intensity images, we found 2,010,671 temporally matched SURF feature measurements, each with a unique timestamp. Assuming a brute-force approach using a discrete-time batch estimator was possible to solve, it would require a pose estimate at each measurement time in the system; meaning that for the $1.1 \mathrm{~km}$ dataset, it would naively require 6 


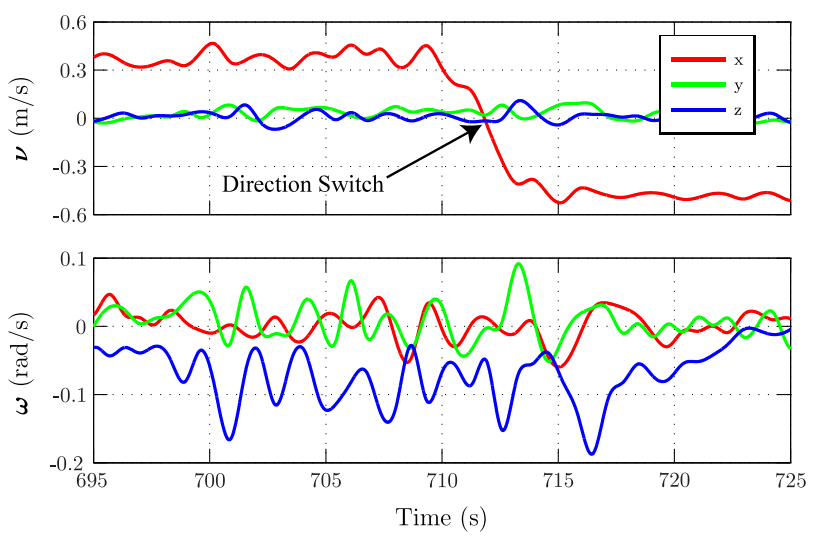

(a) Velocity Profile

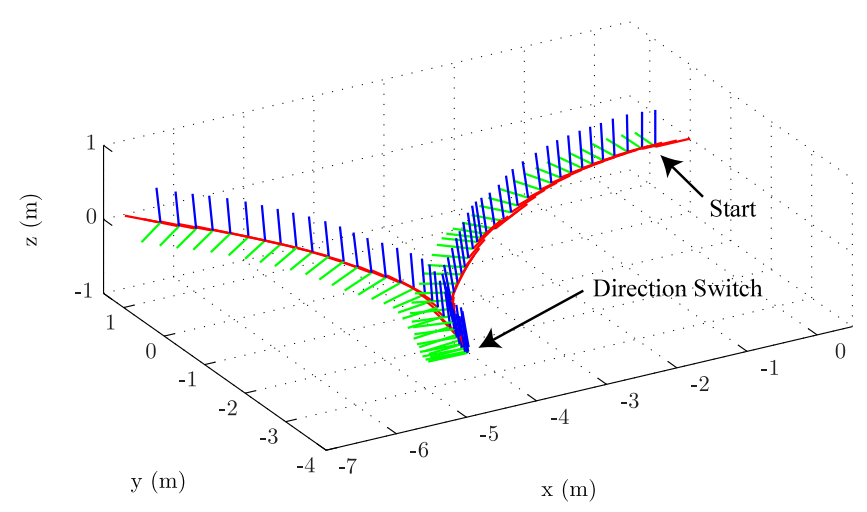

(b) Integrated Trajectory

Figure 18: Example output from the relative continuous-time SLAM estimator using real data. The linear and angular velocity estimates in (a) directly correspond to the integrated 3D trajectory in (b); coordinate frames are plotted at a uniform time interval. The direction switch in forward $(x)$ velocity can be easily observed in the integrated trajectory.

$\times 2,010,671=12,064,026$ state variables, excluding the 688,481 different landmark positions. Using basis functions to parameterize our continuous-time state, we show how the number of required state variables can be drastically reduced.

Before running the constant-time estimator on the entire $1.1 \mathrm{~km}$ trajectory, we must decide on a spacing for the temporal basis functions and an appropriate window size over which to optimize. In order to train these two parameters, we use a $250 \mathrm{~m}$ subsection of the full trajectory. This subsection has 1506 image stacks, from which we extracted SURF features and found 506,675 temporally matched measurements of 169,580 different landmarks. In order to compare the results of the estimator to the GPS ground truth, we consider the integral of our velocity estimate, as seen in Figure 18.

In order to find an appropriate knot spacing, we ran the full batch estimator on the training section a number of times, varying the number of time segments in the B-spline parameterization from 5 to 3000. The accuracy of the solutions are quantified by considering both the total root-mean-square (RMS) Euclidean error, comparing the integrated position of our velocity estimate to the DGPS tracks, and the ALARMS error, for a 10 metre local region. The estimate and DGPS tracks were put into a common reference frame by aligning the first 10 meters of each trajectory.

The result of varying the number of knots can be seen in Figure 19 (timing was broken down into the three major steps described in Section 3.3.8). Using the efficient methods described earlier, we see that the integration and build steps of the algorithm are relatively constant in cost as the number of knots increases. As expected, the solution to $\mathbf{A} \delta \mathbf{z}^{*}=\mathbf{b}$ is cubic in complexity and begins to take a larger portion of the total time as the number of basis functions is increased. As the knot spacing is reduced, the parameterization is given enough degrees of freedom to properly represent the velocity profile of the robot; however, there are diminishing returns with respect to reducing error. For this subsection of the dataset, it was decided that 1200 time segments (a knot spacing of approximately 0.65 seconds) was acceptable to represent the robot trajectory.

In order to limit the growth rate in solve time, we applied our window-style filter to the $250 \mathrm{~m}$ trajectory. In a similar fashion to the first experiment, we then varied the number of active time segments in the optimization window from 1 to 20 . The results in Figure 20 indicate that a window size of only 5 time segments would be sufficient to use over the entire trajectory. The compute time shown in this experiment is the average amount of time taken to solve a single window optimization. Increasing window size has a clear negative effect on the amount of time spent integrating and building the terms needed in the solution.

The final result for the full trajectory, seen in Figure 16, can be compared to the discrete-time relative bundle adjustment implementation, seen in Figure 15. To keep the discrete implementation's state size tractable and solvable, a single relative pose estimate is used for each of the image stacks and the laser scans are assumed to be taken instantaneously (i.e. no motion distortion). The open-loop integration (OLI) of our velocity estimate and discrete relative bundle adjustment solution are put into a common reference frame with the DGPS tracks by aligning the first 30 meters of each trajectory. Compensating for motion distortion by using our continuous-time estimation scheme clearly produces superior results in contrast to the traditional discrete-time approach. 

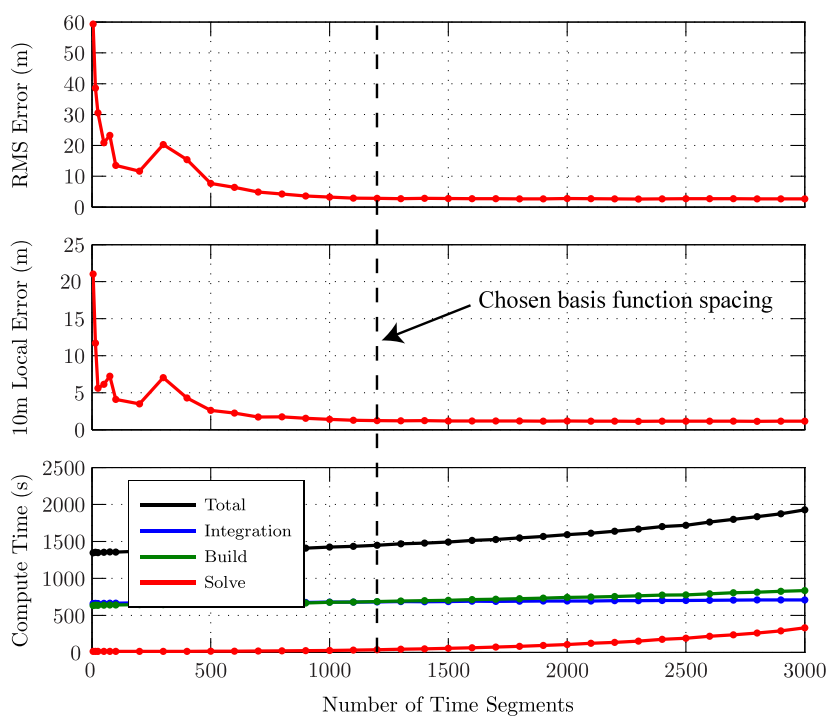

Figure 19: The full batch estimator was run on a $250 \mathrm{~m}$ subsection of the full dataset. Varying the number of time segments in the parameterization from 5 to 3000, we aimed to find an appropriate knot spacing to use over the entire trajectory. It was decided that 1200 time segments (a knot spacing of approximately 0.65 seconds) was acceptable to represent the robot trajectory.
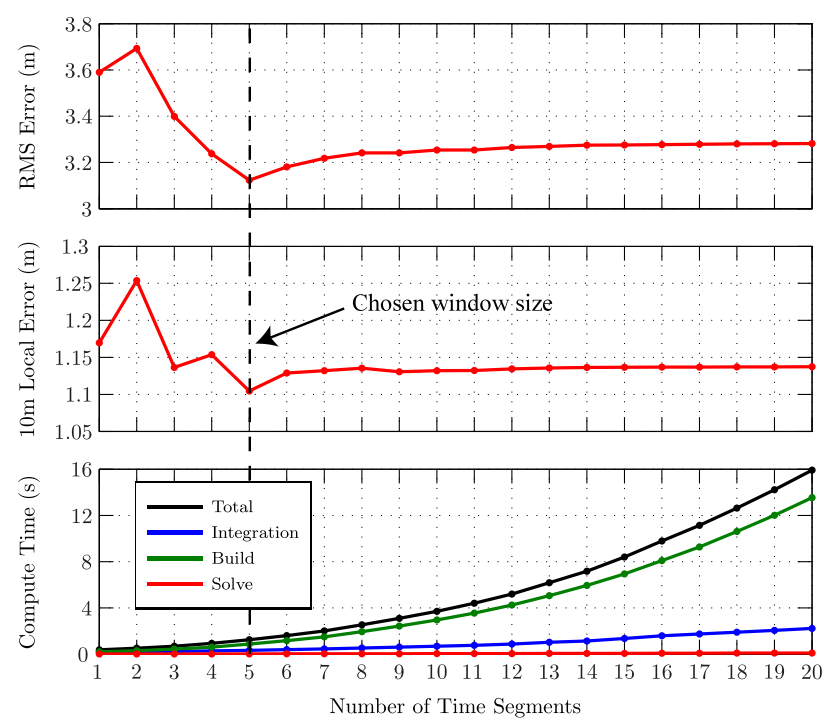

Figure 20: Using 1200 time segments in the B-spline parametrization over the $250 \mathrm{~m}$ training segment, we varied the number of active time segments in each optimization window from 1 to 20 . From these results, the chosen optimization window size for the final experiment was 5 time segments. Here, the compute time is the average time it took to compute a single window optimization.

As suggested by the results of the first experiment, the estimator used a knot spacing of 0.65 seconds. This correlated to the use of only $6 \times 5,386=32,316$ state variables in the parameterization of $\varpi(t)$. In relation to the second experiment, it was decided that the estimator should use a window size of 5 active time segments; this cost approximately 1.3 seconds per local batch optimization and led to a total run time of 1.6 hours ${ }^{1}$.

\subsubsection{Place Recognition}

The visual place-recognition algorithm proposed by MacTavish and Barfoot (2014) was used to detect loop closures. It is based on the FAB-MAP algorithm by Cummins and Newman (2008) and the implementation by Glover et al. (2012). Loop closures are proposed by computing the likelihood that a query intensity image (or group of images) came from each previously visited location. In contrast to the original FAB-MAP algorithm, that compares a single query image to all of the previously seen images using a Bag-of-Words descriptor, the modification proposed by MacTavish and Barfoot (2014) describes and compares groups of images (i.e., they use a bigger bag of words).

FAB-MAP requires a training region to learn a visual vocabulary (quantized SURF features) as well as correlations and occurrence likelihoods for the words in that vocabulary. Figure 21 shows the region of the trajectory that was used for this training, as well as the proposed loop closures. The training region was omitted from the place-recognition image sequence, so no loop closures were considered in this region.

The motion distortion present in the lidar-intensity images makes it difficult to identify places based on appearance. If the path taken through a re-visited location changes the second time through, the images will be distorted differently, thereby changing the observed features. The loops would be much easier to detect if larger sections of the path were repeated while closely following the original path. Instead, the closed loops often have small overlaps and take a slightly different path. In a few occasions, the camera is pointed in the opposite direction, making appearance-based loop closure almost impossible.

Using groups of images allows the algorithm to take advantage of sequence information not strongly used in the single image representation, leading to the performance advantage shown in Figure 22. The Bag-of-Words descriptor is sparsified for image

\footnotetext{
${ }^{1}$ All estimation experiments were implemented in Matlab and timed with a $2.4 \mathrm{GHz}$ processor and $8 \mathrm{~GB}$ of $1333 \mathrm{MHz}$ DDR3 RAM
} 


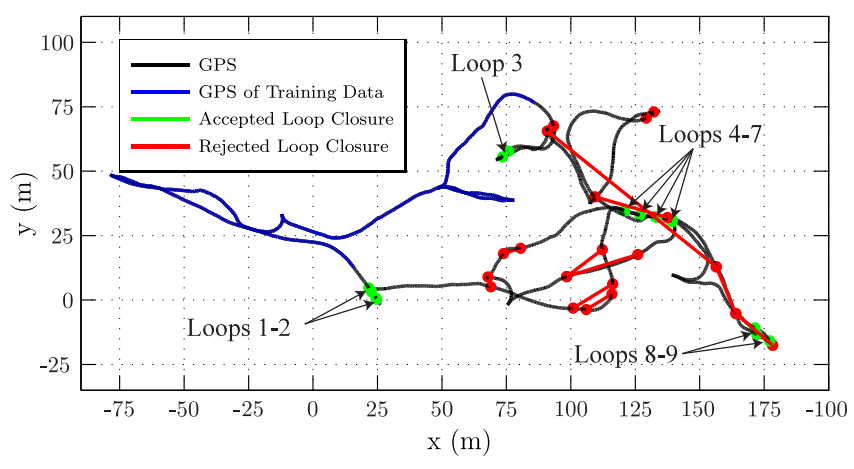

Figure 21: This figure shows the loop closures proposed by the place-recognition algorithm overlaid on GPS data. Place matches were tested geometrically using a rigid RANSAC algorithm; the nine accepted loop closures are shown in green and had over 1000 inlying feature matches, while the rejected loop closures are shown in red. The blue trajectory segment used for training was not evaluated for loop closures.

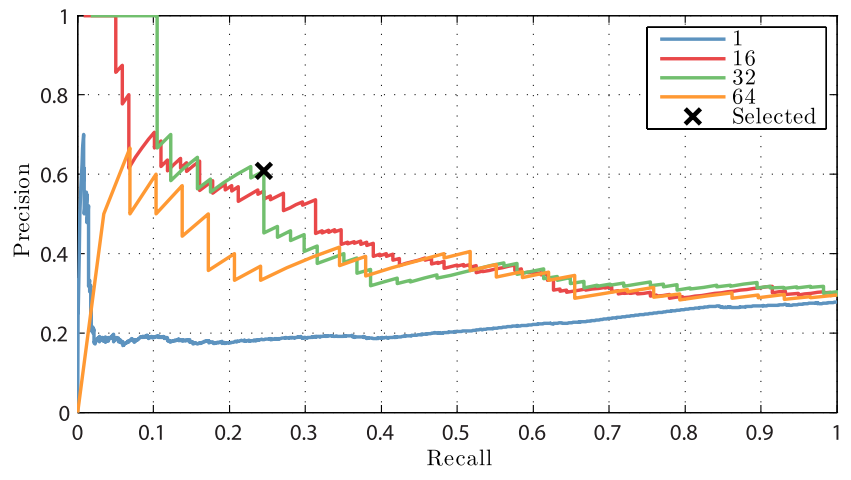

Figure 22: This figure shows the precision-recall curve for the proposed loop closures at different image group sizes. The single-image representation performed very poorly. Matching groups of images improved the proposed loop closures, although it did result in a more costly verification step. For the selected group size of 32 and threshold of $p \geq 0.85$, the precision is $61 \%$ and recall is $25 \%$.

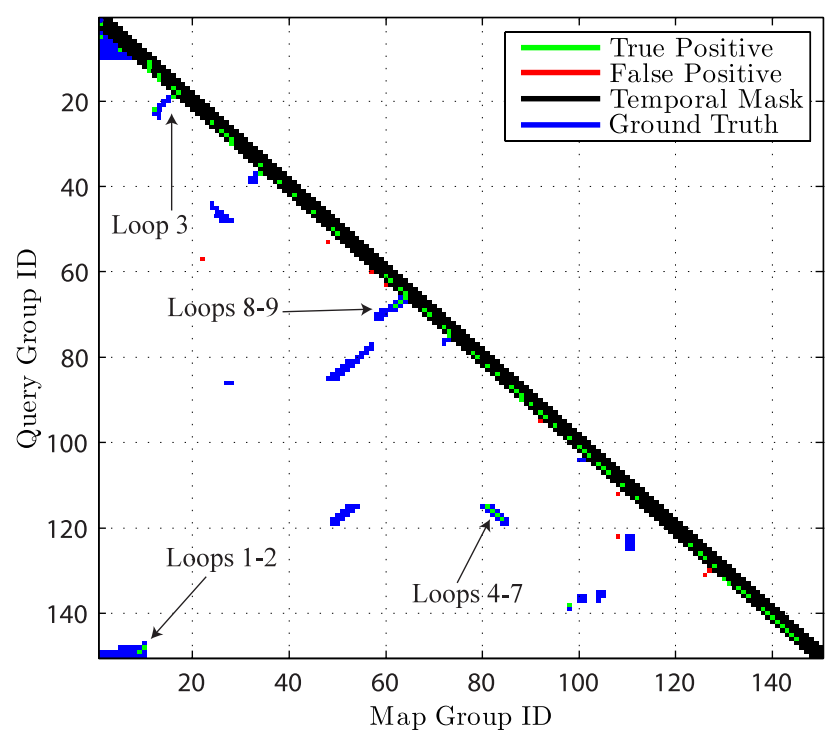

Figure 23: This figure shows an annotated confusion matrix for groups of 32 images, with a probability threshold of $p \geq 0.88$. Image groups temporally close to the query group were masked out. Ground-truth loop closures exist when the GPS locations associated with two images are within $3 \mathrm{~m}$. If any two images across two groups have a loop closure, the two groups inherit that loop closure. The true positives in the bottom-left corner are matches from the end of the trajectory back to the start.

groups, keeping only words commonly observed in the group. These words that have been observed multiple times are likely the more stable features, since they have the same quantization despite having been distorted differently at each observation. Figure 23 is the annotated confusion matrix that shows the matched groups with GPS ground-truth.

In order to geometrically validate the proposed loop closures, the SURF feature measurements from the blocks of 32 images were undistorted into privileged coordinate frames using the relative continuous-time trajectory solution. A traditional rigid RANSAC algorithm was then run to find a transformation that recommends at least 1000 inliers; of the proposed 21 matches, seen in Figure 21, the nine shown in green were accepted and pushed to the estimator.

\subsubsection{Closed-Loop Solution}

At each of the nine loop-closure locations, an optimization was performed for the spatial transformation and the basis functions overlapping in time with spatially matched landmark observations. In order to see how merging landmarks across loop closures improves the open-loop estimate, the solution is integrated using both an OLI and closed-loop integration (CLI) scheme. 


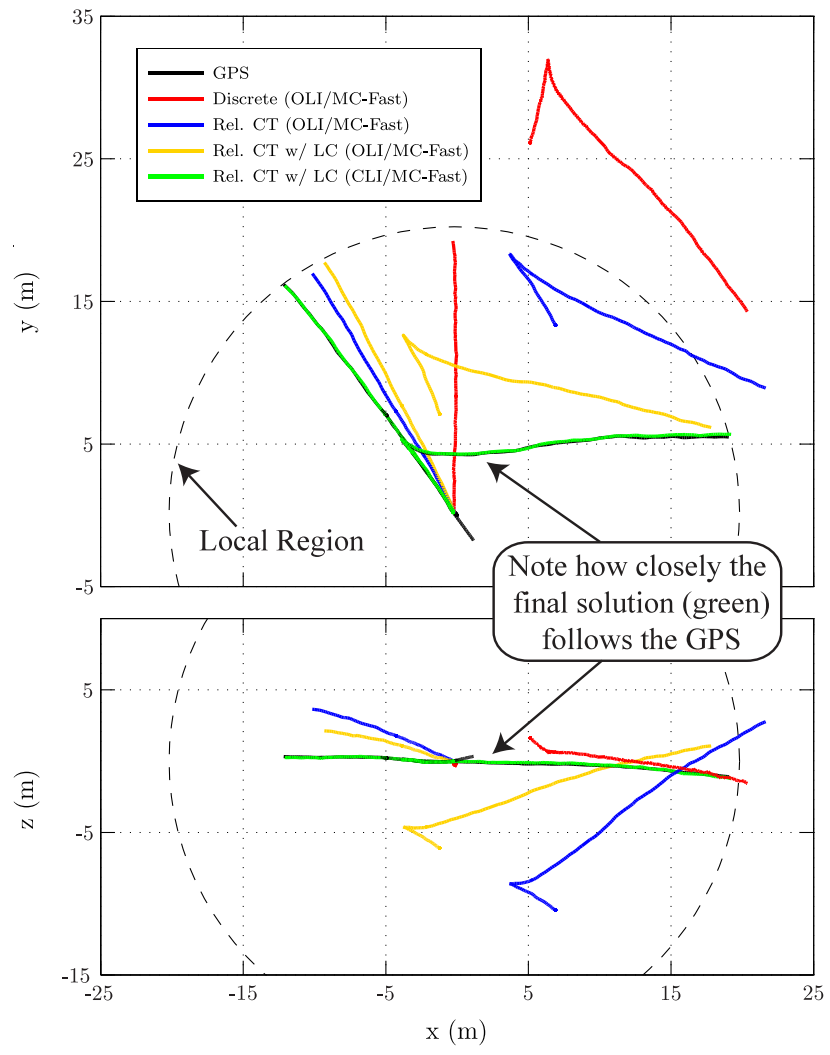

(a) Region 1

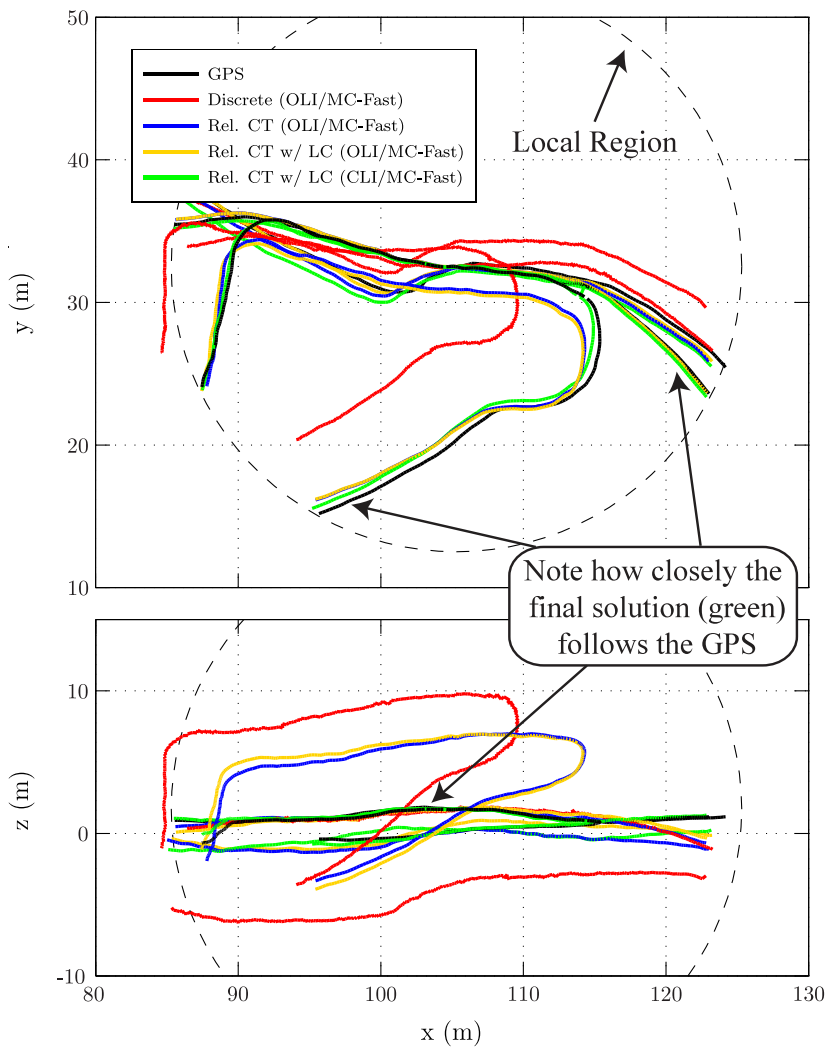

(b) Region 2

Figure 24: These figures show the trajectory integrations for ALARMS error calculations in two different areas; each calculation used a $20 \mathrm{~m}$ local region size and included a loop closure. The first region, shown in (a), is near the start/end of the $1.1 \mathrm{~km}$ trajectory and contains a loop closure connecting the two trajectory segments. An incremental performance increase is clearly visible, with the final solution being very near the GPS ground truth. The second region, shown in (b), includes three trajectory segments, two of which are connected by a loop closure that spans nearly $200 \mathrm{~m}$. Again, an incremental performance increase is clearly visible, however, the final solution is not incredibly accurate due to the lack of loop closure with the third trajectory segment. Loop closure was not possible with the last segment because the robot was facing the opposite direction.

Figure 24 shows the trajectory integrations for two 20 metre local regions, selected as part of ALARMS error calculations, which included loop closures. The first region, in Figure 24(a), includes two trajectory segments, which contain the start and end of the $1.1 \mathrm{~km}$ traversal. The second region, in Figure 24(b), includes three trajectory segments, two of which are connected by a loop closure that spans roughly $200 \mathrm{~m}$. Descriptions of the algorithms and the resultant RMS error for each region is shown in Table 1.

As expected, the discrete algorithm configuration, that does not consider motion distortion, performs poorly in comparison to all of the continuous-time solutions. Note that the integration of the trajectory in the second region included less open-loop distance, and therefore it is expected that the magnitude of errors in the results would be smaller. The expected reduction in error between the open-loop integrations of the relative continuous-time trajectory, with and without loop closure, is fairly minimal. In the first region, we see a fairly substantial improvement, which can be attributed to the small incremental improvements over the very long open-loop integration distance. In the second region, there is a slight increase in measured error; although it is possible that the additional measurements and fusion of landmarks reduced the accuracy of the estimation, it must be considered that the circular error probability (CEP) of our GPS is only $0.4 \mathrm{~m}$ in the horizontal plane. Furthermore, this CEP is reported under ideal conditions and the vertical dimension is typically worst. Finally, we note the solution using CLI in the second region had more than double the error of the first region for a trajectory of similar size; if we consider only the two pieces of trajectory tied by loop closure in the second region, we find the RMS error of the final solution is only 
Table 1: This table describes the different algorithm configurations that will be contrasted for local accuracy. The two estimators are the discrete estimator, that assumes no motion distortion, and the proposed relative continuous-time method. All configurations use the feature correspondences provided by the fast motion-compensated RANSAC algorithm (MC-Fast). The final two configurations take advantage of loop closure information provided by the place-recognition algorithm, but are integrated in different fashions; one using open-loop integration (OLI) and one using closed-loop integration (CLI). Finally, RMS errors are shown for the trajectory integrations investigated in Figure 24.

\begin{tabular}{|l|l|l|l|l|l|l|}
\hline Label & Estimator & $\begin{array}{l}\text { Outlier } \\
\text { Rejection }\end{array}$ & $\begin{array}{l}\text { Loop } \\
\text { Closures }\end{array}$ & $\begin{array}{l}\text { Integration } \\
\text { Scheme }\end{array}$ & $\begin{array}{l}\text { RMS Error } \\
\text { in Region 1 }\end{array}$ & $\begin{array}{l}\text { RMS Error } \\
\text { in Region 2 }\end{array}$ \\
\hline Discrete (OLI/MC-Fast) & Discrete & MC-Fast & No & OLI & $13.95 \mathrm{~m}$ & $6.32 \mathrm{~m}$ \\
\hline Rel. CT (OLI/MC-Fast) & Relative CT & MC-Fast & No & OLI & $8.61 \mathrm{~m}$ & $2.83 \mathrm{~m}$ \\
\hline Rel. CT w/ LC (OLI/MC-Fast) & Relative CT & MC-Fast & Yes & OLI & $4.41 \mathrm{~m}$ & $2.89 \mathrm{~m}$ \\
\hline Rel. CT w/ LC (CLI/MC-Fast) & Relative CT & MC-Fast & Yes & CLI & $0.40 \mathrm{~m}$ & $1.14 \mathrm{~m}$ \\
\hline
\end{tabular}

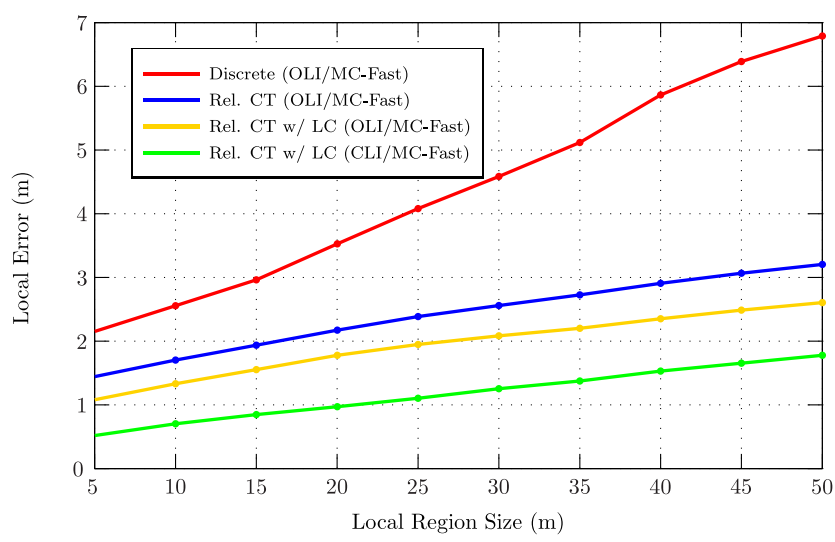

Figure 25: This figure shows ALARMS error for a number of local region sizes, using trajectory estimates of the entire $1.1 \mathrm{~km}$ trajectory. Note the estimation error drift for continuous-time solutions is far lower than that of the discrete solution. Furthermore, the addition of loop closure improves both the open-loop and closed-loop results.

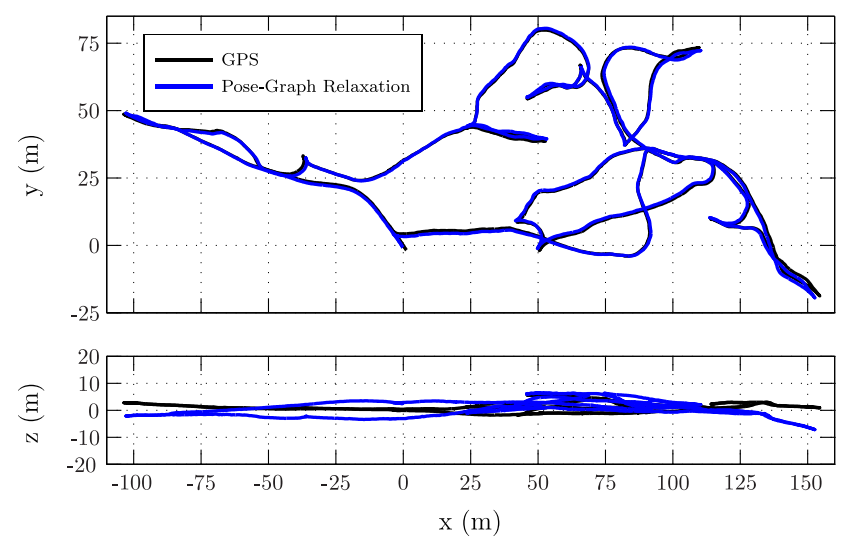

Figure 26: This figure shows the globally consistent trajectory estimate generated by discretizing the full $1.1 \mathrm{~km}$ continuous-time trajectory, and performing a pose-graph relaxation using the nine available loop closures. The RMS error for the full trajectory is $2.9 \mathrm{~m}$; considering only the $\mathrm{x}-\mathrm{y}$ plane, the RMS error is $0.77 \mathrm{~m}$.

$0.44 \mathrm{~m}$, giving some insight to the importance of place recognition for increasing local estimation accuracy. We contrast the various algorithm configurations more broadly, over the full $1.1 \mathrm{~km}$ trajectory, by comparing the ALARMS error for varying local region sizes, as seen in Figure 25. Lastly, we note that the number of available loop closures in this dataset was fairly low, but that the algorithm should scale well with additional loop closures; Sibley et al. (2010) show in their discrete-time relative bundle adjustment algorithm that the addition of many loop closures significantly reduces mapping error, even before global relaxation.

In an offline process, we discretized the continuous-time trajectory, solved for the covariance of the relative transforms, and performed pose-graph relaxation using the nine optimized loop closures. The resulting globally consistent trajectory estimate can be seen in Figure 26. While a globally consistent map of this quality is useful for tasks such as macro-level path planning, or low-frequency human-in-the-loop decisions, we reiterate that the strength of the relative formulation is a constant-time, locally accurate solution aimed at enabling autonomous tasks, such as navigation or object avoidance.

Lastly, in Figure 27, we show a locally consistent point cloud generated by reprojecting dense lidar data from the integrated continuous-time pose in an area involving loop closure. From a navigational standpoint, the ability to close loops in constant time and generate highly accurate local maps is significant; especially with the growing popularity of techniques that aim to localize against previously traversed paths in order to repeat routes (Furgale and Barfoot, 2010; Churchill and Newman, 2012). 


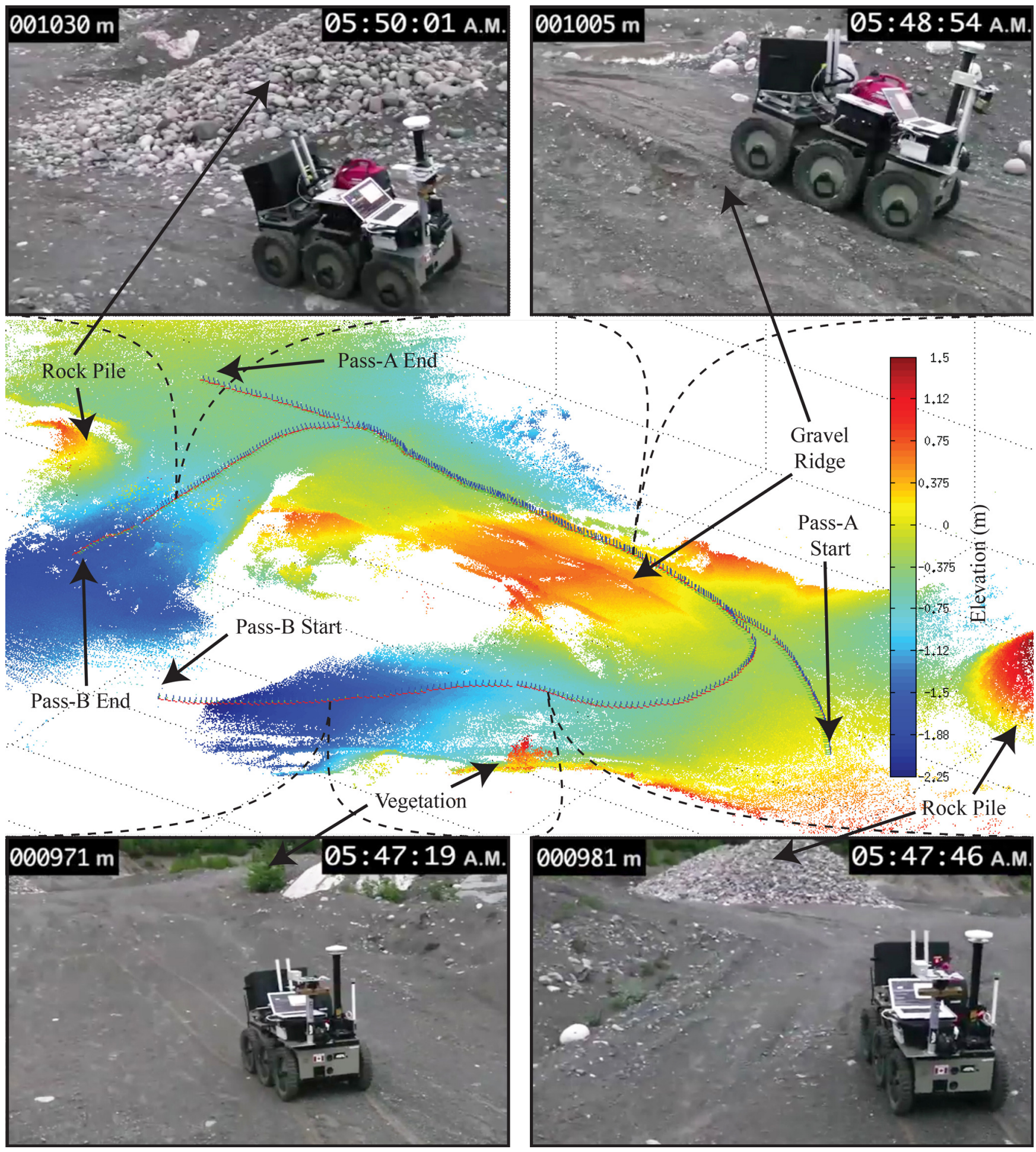

Figure 27: This figure shows reprojected dense lidar data, coloured by elevation, from a local region of our experiment that included loop closure, and demonstrates the ability of our algorithm to produce locally accurate and consistent maps. Furthermore, it attests to the types of unstructured terrain we traversed with a scanning lidar sensor. Photographs of the experiment are used to provide context and the sensor's estimated coordinate frame is shown at the nominal frame times. 


\section{Conclusion}

This paper investigates the use of a scanning sensor in the visual-motion-estimation pipeline. We developed novel methods to perform both outlier rejection and incremental, relative batch optimizations with motion-distorted data. A novel adaptation of the traditional RANSAC algorithm was developed using both the geometric and temporal measurement data to solve for the 6D constant velocity of the sensor. To improve computational performance, this paper also proposes a Euclidean least-squares estimator and a heuristic for the point-transformation step. To generate a more accurate trajectory estimate, a novel estimation scheme was derived by reformulating relative SLAM in continuous time. Using temporal basis functions with local support to parameterize the velocity profile of the robot, we are able to apply a window-style filter to incrementally solve the SLAM problem with local accuracy in constant time (even at loop closure). The two proposed algorithms were validated using a 6880 frame sequence of lidar intensity and range imagery, captured over a $1.1 \mathrm{~km}$ trajectory. A local error metric was used to quantify the ability of our system to generate locally accurate trajectory estimates, and dense point cloud data were reprojected from two local regions of our closed-loop solution to show the creation of high quality maps.

\section{Funding}

This research received no specific grant from any funding agency in the public, commercial, or not-for-profit sectors.

\section{A Minimal Point Set to Estimate a Constant Velocity}

Following from the derivation of our nonlinear motion-compensated RANSAC algorithm, in Section 4.3.1, the goal of this section is to derive the conditions under which we can solve (85) for a six-dimensional constant velocity. In order to not overcomplicate the derivation, we assume the use of Euclidean point measurements, defining our camera model as,

$$
\mathbf{f}(\mathbf{v}):=\mathbf{v}
$$

and using an isotropic covariance,

$$
\mathbf{R}_{m, 2}=\sigma^{2} \mathbf{1}
$$

Using these simplifying assumptions, the system of equations in (85), becomes,

$$
\left(\sum_{m} \mathbf{G}_{m}^{T} \mathbf{G}_{m}\right) \delta \varpi^{*}=\sum_{m} \mathbf{G}_{m}^{T}\left(\mathbf{p}_{m, 2}-\mathbf{g}_{m}\right),
$$

correct to first order, where

$$
\begin{aligned}
\mathbf{g}_{m} & :=\overline{\mathbf{T}}_{m} \mathbf{p}_{m, 1}=\exp \left(\Delta t_{m} \bar{\varpi}^{\wedge}\right) \mathbf{p}_{m, 1}, \\
\mathbf{G}_{m} & :=\Delta t_{m} \mathbf{g}_{m}^{\odot} \mathcal{J}\left(\Delta t_{m} \bar{\varpi}\right),
\end{aligned}
$$

and the points, $\mathbf{p}_{m, 1}$ and $\mathbf{p}_{m, 2}$, are matched point measurements, the temporal difference between measurements is $\Delta t_{m}$, and $\bar{\varpi}$ is the nominal constant-velocity estimate. In an iterative fashion, the nominal solution is updated, using $\bar{\varpi} \leftarrow \bar{\varpi}+\delta \varpi^{*}$, and will converge to a local minimum determined by the shape of the objective function and the initial condition.

At this point in the derivation, we require a simplification in order to show the conditions under which the system is solvable. Rather than make the somewhat harsh assumption that the product $\Delta t_{m} \bar{\varpi}$ is small, such that $\overline{\mathbf{T}}_{m} \approx \mathbf{1}$ and $\mathcal{J}\left(\Delta t_{m} \bar{\varpi}\right) \approx \mathbf{1}$, as we did in the fast and approximate implementation of our motion-compensated RANSAC algorithm, we instead make the less restrictive assumption that $\Delta t_{m} \bar{\varpi}$ is small enough, such that,

$$
\mathcal{J}\left(\Delta t_{m} \bar{\varpi}\right) \approx \mathcal{T}\left(\frac{1}{2} \Delta t_{m} \bar{\varpi}\right)
$$

where the functions $\mathcal{J}(\boldsymbol{\xi})$ and $\mathcal{T}\left(\frac{1}{2} \boldsymbol{\xi}\right)$ can be computed according to (Barfoot and Furgale, 2014),

$$
\begin{gathered}
\mathcal{J}(\boldsymbol{\xi})=\sum_{n=0}^{\infty} \frac{1}{(n+1) !}\left(\boldsymbol{\xi}^{\curlywedge}\right)^{n}=\mathbf{1}+\frac{1}{2} \boldsymbol{\xi}^{\curlywedge}+\frac{1}{6} \boldsymbol{\xi}^{\curlywedge} \boldsymbol{\xi}^{\curlywedge}+\frac{1}{24} \boldsymbol{\xi}^{\curlywedge} \boldsymbol{\xi}^{\curlywedge} \boldsymbol{\xi}^{\curlywedge}+\ldots, \\
\mathcal{T}\left(\frac{1}{2} \boldsymbol{\xi}\right)=\sum_{n=0}^{\infty} \frac{1}{n !}\left(\frac{1}{2} \boldsymbol{\xi}^{\curlywedge}\right)^{n}=\mathbf{1}+\frac{1}{2} \boldsymbol{\xi}^{\curlywedge}+\frac{1}{8} \boldsymbol{\xi}^{\curlywedge} \boldsymbol{\xi}^{\curlywedge}+\frac{1}{48} \boldsymbol{\xi}^{\curlywedge} \boldsymbol{\xi}^{\curlywedge} \boldsymbol{\xi}^{\curlywedge}+\ldots
\end{gathered}
$$


The assumption is correct for terms in $\mathbf{G}_{m}^{T} \mathbf{G}_{m}$ up to order $\Delta t_{m}^{3}$, and a good approximation thereafter for small products of $\Delta t_{m} \bar{\varpi}$. In combination with the identity in (48b), we then find that

$$
\mathbf{G}_{m} \approx \Delta t_{m} \exp \left(\frac{1}{2} \Delta t_{m} \bar{\varpi}^{\wedge}\right)\left(\exp \left(\frac{1}{2} \Delta t_{m} \overline{\boldsymbol{\varpi}}^{\wedge}\right) \mathbf{p}_{m, 1}\right)^{\odot}
$$

It follows that

$$
\sum_{m} \mathbf{G}_{m}^{T} \mathbf{G}_{m} \approx \mathcal{M}
$$

where the $6 \times 6$ matrix, $\mathcal{M}$, has the form of a generalized mass matrix (Murray et al., 1994),

$$
\mathcal{M}:=\sum_{m} \Delta t_{m}^{2} \mathbf{h}_{m}^{\odot}{ }^{T} \mathbf{h}_{m}^{\odot}=\left[\begin{array}{cc}
\mathbf{1} & \mathbf{0} \\
-w \mathbf{r}^{\wedge} & \mathbf{1}
\end{array}\right]\left[\begin{array}{cc}
w \mathbf{1} & \mathbf{0} \\
\mathbf{0} & \mathbf{I}
\end{array}\right]\left[\begin{array}{cc}
\mathbf{1} & w \mathbf{r}^{\wedge} \\
\mathbf{0} & \mathbf{1}
\end{array}\right]
$$

where

$$
\begin{aligned}
\mathbf{h}_{m} & =\left[\begin{array}{c}
\mathbf{r}_{m} \\
1
\end{array}\right]=\exp \left(\frac{1}{2} \Delta t_{m} \bar{\varpi}^{\wedge}\right) \mathbf{p}_{m, 1}, \quad w=\sum_{m} \Delta t_{m}^{2}, \\
\mathbf{r} & =\frac{1}{w} \sum_{m} \Delta t_{m}^{2} \mathbf{r}_{m}, \quad \mathbf{I}=-\sum_{m} \Delta t_{m}^{2}\left(\mathbf{r}_{m}-\mathbf{r}\right)^{\wedge}\left(\mathbf{r}_{m}-\mathbf{r}\right)^{\wedge} .
\end{aligned}
$$

With respect to the conditions under which (93) has a unique solution, it follows from (99) that $\operatorname{det} \boldsymbol{M}=w \operatorname{det} \mathbf{I}$. Given that $\Delta t_{m}^{2}>0$, we know $w>0$, and therefore the unique solution of $\delta \varpi$ requires only that $\operatorname{det} \mathbf{I} \neq \mathbf{0}$. For this to be true, it is sufficient to show that I is positive definite, which implies that for any $\mathbf{x} \neq \mathbf{0}$, we have that $\mathbf{x}^{T} \mathbf{I} \mathbf{x}>\mathbf{0}$. Examining the structure of $\mathbf{I}$, we find that

$$
\mathbf{x}^{T} \mathbf{I} \mathbf{x}=-\mathbf{x}^{T} \sum_{m} \Delta t_{m}^{2}\left(\mathbf{r}_{m}-\mathbf{r}\right)^{\wedge}\left(\mathbf{r}_{m}-\mathbf{r}\right)^{\wedge} \mathbf{x}=\sum_{m} \Delta t_{m}^{2} \underbrace{\left(\left(\mathbf{r}_{m}-\mathbf{r}\right)^{\wedge} \mathbf{x}\right)^{T}\left(\left(\mathbf{r}_{m}-\mathbf{r}\right)^{\wedge} \mathbf{x}\right)}_{\geq 0} \geq 0 .
$$

Given that all the terms in the summation are non-negative, the total must also be non-negative. In order for the total to be zero, each term of the summation must be zero, meaning that for each $m$, either $\mathbf{r}_{m}=\mathbf{r}, \mathbf{x}$ is parallel to $\mathbf{r}_{m}-\mathbf{r}$, or $\mathbf{x}=\mathbf{0}$. Based on the first two cases (the last case is not true by assumption), the sufficient conditions to ensure a unique solution is that there are a minimum of three points, and that the three points are not collinear. In contrast to a typical rigid formulation, we note that this non-collinearity constraint happens in a virtual reference frame, into which the points $\mathbf{p}_{m, 1}$ are transformed based on the current velocity estimate, $\bar{\varpi}$, and the different time intervals, $\Delta t_{m}$; interestingly, this means that the system could be solvable using three points that are collinear in world space, given a non-zero sensor velocity.

\section{References}

Ait-Aider, O., Andreff, N., Lavest, J., and Martinet, P. (2006). Simultaneous object pose and velocity computation using a single view from a rolling shutter camera. Computer Vision-ECCV 2006, pages 56-68.

Ait-Aider, O. and Berry, F. (2009). Structure and kinematics triangulation with a rolling shutter stereo rig. In Computer Vision, 2009 IEEE 12th International Conference on, pages 1835-1840. IEEE.

Anderson, S. and Barfoot, T. D. (2013a). Ransac for motion-distorted 3d visual sensors. In IEEE/RSJ International Conference on Intelligent Robots and Systems (IROS), Tokyo, Japan.

Anderson, S. and Barfoot, T. D. (2013b). Towards relative continuous-time slam. In Proceedings of the IEEE International Conference on Robotics and Automation (ICRA), Karlsruhe, Germany.

Anderson, S., Dellaert, F., and Barfoot, T. D. (2014). A hierarchical wavelet decomposition for continuous-time slam. In Proceedings of the IEEE International Conference on Robotics and Automation (ICRA), Hong Kong, China.

Anderson, S., McManus, C., Dong, H., Beerepoot, E., and Barfoot, T. D. (2012). The gravel pit lidar-intensity imagery dataset. Technical Report ASRL-2012-ABL001, UTIAS. 
Arun, K. S., Huang, T. S., and Blostein, S. D. (1987). Least-squares fitting of two 3-d point sets. Pattern Analysis and Machine Intelligence, IEEE Transactions on, (5):698-700.

Barfoot, T. D. and Furgale, P. T. (2014). Associating uncertainty with three-dimensional poses for use in estimation problems. IEEE Transactions on Robotics.

Barfoot, T. D., McManus, C., Anderson, S., Dong, H., Beerepoot, E., Tong, C. H., Furgale, P., Gammell, J. D., and Enright, J. (2013). Into darkness: Visual navigation based on a lidar-intensity-image pipeline. In Proceedings of the International Symposium on Robotics Research (ISRR), Singapore.

Barfoot, T. D., Tong, C. H., and Särkkä, S. (2014). Batch continuous-time trajectory estimation as exactly sparse gaussian process regression. In Proceedings of Robotics: Science and Systems (RSS), Berkeley, USA.

Bay, H., Tuytelaars, T., and Van Gool, L. (2006). Surf: Speeded up robust features. Computer Vision-ECCV 2006, pages 404-417.

Bibby, C. and Reid, I. (2010). A hybrid slam representation for dynamic marine environments. In Proceedings of the IEEE International Conference on Robotics and Automation (ICRA), pages 257-264. IEEE.

Bosse, M. and Zlot, R. (2009). Continuous 3d scan-matching with a spinning 2d laser. In Proceedings of the IEEE International Conference on Robotics and Automation (ICRA), pages 4312-4319. IEEE.

Bosse, M., Zlot, R., and Flick, P. (2012). Zebedee: Design of a spring-mounted 3-d range sensor with application to mobile mapping. IEEE Transactions on Robotics, 28(5):1104-1119.

Brown, D. (1958). A solution to the general problem of multiple station analytical stereo-triangulation. rca data reduction. Technical report.

Churchill, W. and Newman, P. (2012). Practice makes perfect? managing and leveraging visual experiences for lifelong navigation. In Proceedings of the IEEE International Conference on Robotics and Automation, pages 4525-4532. IEEE.

Cummins, M. and Newman, P. (2008). Fab-map: Probabilistic localization and mapping in the space of appearance. The International Journal of Robotics Research, 27(6):647-665.

Dellaert, F. and Kaess, M. (2006). Square root sam: Simultaneous localization and mapping via square root information smoothing. The International Journal of Robotics Research, 25(12):1181-1203.

Dong, H. and Barfoot, T. D. (2012). Lighting-invariant visual odometry using lidar intensity imagery and pose interpolation. In Proceedings of the International Conference on Field and Service Robotics (FSR), Matsushima, Japan.

Fischler, M. and Bolles, R. (1981). Random sample consensus: a paradigm for model fitting with applications to image analysis and automated cartography. Communications of the ACM, 24(6):381-395.

Fleps, M., Mair, E., Ruepp, O., Suppa, M., and Burschka, D. (2011). Optimization based imu camera calibration. In In Proceedings of the IEEE International Conference on Intelligent Robots and Systems (IROS), pages 3297-3304.

Furgale, P. T. and Barfoot, T. D. (2010). Visual teach and repeat for long-range rover autonomy. Journal of Field Robotics, special issue on "Visual mapping and navigation outdoors", 27(5):534-560.

Furgale, P. T., Barfoot, T. D., and Sibley, G. (2012). Continuous-time batch estimation using temporal basis functions. In Proceedings of the IEEE International Conference on Robotics and Automation (ICRA), pages 2088-2095, St. Paul, USA.

Glover, A., Maddern, W., Warren, M., Reid, S., Milford, M., and Wyeth, G. (2012). Openfabmap: An open source toolbox for appearance-based loop closure detection. In IEEE International Conference on Robotics and Automation, 2012, pages 4730-4735. IEEE.

Grisetti, G., Stachniss, C., Grzonka, S., and Burgard, W. (2007). A tree parameterization for efficiently computing maximum likelihood maps using gradient descent. In Proceedings of Robotics: Science and Systems, Atlanta, GA, USA. 
Haralick, B. M., Lee, C.-N., Ottenberg, K., and Nölle, M. (1994). Review and analysis of solutions of the three point perspective pose estimation problem. International Journal of Computer Vision, 13(3):331-356.

Hedborg, J., Forssén, P., Felsberg, M., and Ringaby, E. (2012). Rolling shutter bundle adjustment. In IEEE Conference on Computer Vision and Pattern Recognition, pages 1434-1441.

Hedborg, J., Ringaby, E., Forssén, P.-E., and Felsberg, M. (2011). Structure and motion estimation from rolling shutter video. In Computer Vision Workshops (ICCV Workshops), 2011 IEEE International Conference on, pages 17-23. IEEE.

Horn, B. K. (1987). Closed-form solution of absolute orientation using unit quaternions. JOSA A, 4(4):629-642.

Jia, C. and Evans, B. L. (2012). Probabilistic 3-d motion estimation for rolling shutter video rectification from visual and inertial measurements. In Proc. IEEE Int. Workshop on Multimedia Signal Processing.

Kim, M.-J., Kim, M.-S., and Shin, S. Y. (1995). A general construction scheme for unit quaternion curves with simple high order derivatives. In Proceedings of the 22nd annual conference on Computer graphics and interactive techniques, pages 369-376. ACM.

Konolige, K., Bowman, J., Chen, J., Mihelich, P., Calonder, M., Lepetit, V., and Fua, P. (2010). View-based maps. The International Journal of Robotics Research, 29(8):941.

Lovegrove, S., Patron-Perez, A., and Sibley, G. (2013). Spline fusion: A continuous-time representation for visual-inertial fusion with application to rolling shutter cameras. British Machine Vision Conference (BMVC13).

Lucas, B. D. and Kanade, T. (1981). An iterative image registration technique with an application to stereo vision. In Proceedings of the 7th international joint conference on Artificial intelligence.

MacTavish, K. and Barfoot, T. D. (2014). Towards hierarchical place recognition for long-term autonomy. In Proceedings of the IEEE International Conference on Robotics and Automation workshop on "Visual Place Recognition in Changing Environments", Hong Kong, China.

Maimone, M., Cheng, Y., and Matthies, L. (2007). Two years of visual odometry on the mars exploration rovers. Journal of Field Robotics, 24(3):169-186.

McManus, C., Furgale, P. T., and Barfoot, T. D. (2011). Towards appearance-based methods for lidar sensors. In Proceedings of the IEEE International Conference on Robotics and Automation (ICRA), pages 1930-1935, Shanghai, China.

McManus, C., Furgale, P. T., and Barfoot, T. D. (2013). Towards lighting-invariant visual navigation: An appearance-based approach using scanning laser-rangefinders. Robotics and Autonomous Systems, 61:836-852.

Murray, R. M., Li, Z., and Sastry, S. (1994). A mathematical introduction to robotic manipulation. Boca Raton, FL: CRC.

Nistér, D. (2004). An efficient solution to the five-point relative pose problem. Pattern Analysis and Machine Intelligence, IEEE Transactions on, 26(6):756-770.

Olson, E., Leonard, J., and Teller, S. (2006). Fast iterative alignment of pose graphs with poor initial estimates. In Proceedings of the IEEE International Conference on Robotics and Automation, pages 2262-2269. IEEE.

Oth, L., Furgale, P. T., Kneip, L., and Siegwart, R. (2013). Rolling shutter camera calibration. In Proc. of The IEEE International Conference on Computer Vision and Pattern Recognition (CVPR), Portland, USA.

Sibley, G., Mei, C., Reid, I., and Newman, P. (2009). Adaptive relative bundle adjustment. In Proceedings of Robotics: Science and Systems, Seattle, USA.

Sibley, G., Mei, C., Reid, I., and Newman, P. (2010). Vast-scale outdoor navigation using adaptive relative bundle adjustment. The International Journal of Robotics Research, 29(8):958.

Thrun, S., Burgard, W., and Fox, D. (2005). Probabilistic Robotics (Intelligent Robotics and Autonomous Agents). The MIT Press. 
Tong, C., Anderson, S., Dong, H., and Barfoot, T. D. (2014). Pose interpolation for laser-based visual odometry. Journal of Field Robotics, special issue on "Field and Service Robotics", 31(5):787-813.

Tong, C. H., Furgale, P. T., and Barfoot, T. D. (2013). Gaussian process gauss-newton for non-parametric simultaneous localization and mapping. International Journal of Robotics Research, 32(5):507-525.

Triggs, B., McLauchlan, P., Hartley, R., and Fitzgibbon, A. (2000). Bundle adjustment - a modern synthesis. Vision algorithms: theory and practice, pages 153-177.

Umeyama, S. (1991). Least-squares estimation of transformation parameters between two point patterns. IEEE Transactions on pattern analysis and machine intelligence, 13(4):376-380.

Zlot, R. and Bosse, M. (2012). Efficient large-scale 3d mobile mapping and surface reconstruction of an underground mine. In Proceedings of the International Conference on Field and Service Robotics (FSR), Matsushima, Japan.

Zlot, R. and Bosse, M. (2014). Efficient large-scale three-dimensional mobile mapping for underground mines. Journal of Field Robotics. 